manuscript No.

(will be inserted by the editor)

\title{
Truncated linear statistics associated with the top eigenvalues of random matrices
}

\author{
Aurélien Grabsch • Satya N. Majumdar • \\ Christophe Texier
}

May 16, 2018

\begin{abstract}
Given a certain invariant random matrix ensemble characterised by the joint probability distribution of eigenvalues $P\left(\lambda_{1}, \ldots, \lambda_{N}\right)$, many important questions have been related to the study of linear statistics of eigenvalues $L=\sum_{i=1}^{N} f\left(\lambda_{i}\right)$, where $f(\lambda)$ is a known function. We study here truncated linear statistics where the sum is restricted to the $N_{1}<N$ largest eigenvalues: $\tilde{L}=\sum_{i=1}^{N_{1}} f\left(\lambda_{i}\right)$. Motivated by the analysis of the statistical physics of fluctuating one-dimensional interfaces, we consider the case of the Laguerre ensemble of random matrices with $f(\lambda)=\sqrt{\lambda}$. Using the Coulomb gas technique, we study the $N \rightarrow \infty$ limit with $N_{1} / N$ fixed. We show that the constraint that $\tilde{L}=\sum_{i=1}^{N_{1}} f\left(\lambda_{i}\right)$ is fixed drives an infinite order phase transition in the underlying Coulomb gas. This transition corresponds to a change in the density of the gas, from a density defined on two disjoint intervals to a single interval. In this latter case the density presents a logarithmic divergence inside the bulk. Assuming that $f(\lambda)$ is monotonous, we show that these features arise for any random matrix ensemble and truncated linear statitics, which makes the scenario described here robust and universal.
\end{abstract}

PACS numbers : 05.40.-a ; 02.50.-r ; 05.70.Np

\section{Introduction}

Introduced in physics by Wigner and Dyson in the 1950s in order to model the complexity in atomic nucleus, random matrix theory has irrigated many fields of physics, ranging from electronic quantum transport [1-13], quantum information (entanglement in random bipartite quantum states) [14-19] or statistical physics of fluctuating interfaces [20,21] (see [22] for a review). The

Aurélien Grabsch · Satya N. Majumdar · Christophe Texier

LPTMS, CNRS, Univ. Paris-Sud, Université Paris-Saclay, 91405 Orsay, France 
first questions arising in nuclear physics were related to the statistical analysis of the spectrum (level distribution and correlations), encoded in the joint probability density function of the eigenvalues $P\left(\lambda_{1}, \ldots, \lambda_{N}\right)$ characterizing the matrix ensemble. Another class of questions has arisen later, concerning the statistical properties of linear statistics of the eigenvalues

$$
L=\sum_{n=1}^{N} f\left(\lambda_{n}\right)
$$

where $f$ is a given function (not necessarily linear). Many physical quantities can be expressed under such a form, as illustrated in the aforementioned references. Several tools have been developed to tackle this problem within invariant random matrix ensembles, ${ }^{1}$ such as orthogonal polynomials, Selberg's integrals or the Coulomb gas method. Although orthogonal polynomials method can be used to compute the moments of $L$ and yields explicit formulae for the characteristic function in terms of determinants, these results are quite difficult to use in practice, in particular to study the limit $N \rightarrow \infty$. In this limit, the Coulomb gas technique proves to be the most efficient method for the analysis of the full distribution (in particular the large deviation tails characterizing the atypical fluctuations). The idea is to interpret the eigenvalue distribution $P\left(\lambda_{1}, \ldots, \lambda_{N}\right)$ as the Gibbs measure for a one-dimensional (1D) gas of particles, "charges", with logarithmic interactions (eigenvalues then correspond to the positions of the particles) [23]. For references in the mathematical literature, cf. [24,25]. The analysis of the distribution of the linear statistics $L$ is then mapped onto the determination of the configuration of charges that minimizes the energy of the gas under the constraint that $L=\sum_{n} f\left(\lambda_{n}\right)$ is fixed. In the thermodynamic limit, $N \rightarrow \infty$, the density of eigenvalues can be considered as continuous, which makes the optimization problem solvable by several techniques such as by resolvent method [26] or using the Tricomi theorem [27]. This Tricomi's theorem was found very useful and was first used to obtain the large deviation function associated to the distribution of the largest eigenvalue, say $\lambda_{1}$, of Gaussian matrices [27,28] and Wishart matrices [29]. This problem can be related to the study of a linear statistics of the form (1.1) as the cumulative distribution of the largest eigenvalue coincides with the probability that all the eigenvalues are below the threshold, thus $\operatorname{Proba}\left\{\lambda_{1} \leqslant W\right\}=\operatorname{Proba}\{L=N\}$ for $f(\lambda)=\Theta(W-\lambda)$, where $\Theta$ is the Heaviside step-function. The problem has been further generalised to consider the number of eigenvalues in an arbitrary interval, $f(\lambda)=\mathbf{1}_{[a, b]}(\lambda)$ [30-32], a question relevant in various contexts, like principal component analysis in statistics [33] or particle-number fluctuations of fermions in a harmonic trap at zero temperature $[34,35]$.

An interesting aspect of the statistical analysis of linear statistics is the possibility of phase transitions in the Coulomb gas, driven by the constraint,

1. The case where the matrix distribution is invariant under changes of basis, i.e. when eigenvalues and eigenvectors are uncorrelated. 
which correspond to transitions in the density of the optimal charge configuration: for example the splitting of the density or the transition between a soft edge and a hard edge (density vanishing or diverging at a boundary). Several examples were studied in Refs. [8,10,12,13,18-20,22,36] (see also Table 5.1 in the conclusion).

In this paper, we consider a new kind of constraint arising from the study of linear statistics restricted to the $N_{1}<N$ largest eigenvalues:

$$
\tilde{L}=\sum_{n=1}^{N_{1}} f\left(\lambda_{n}\right) \quad \text { with } \lambda_{1}>\lambda_{2}>\cdots>\lambda_{N} .
$$

This new problem interpolates between the two important types of questions mentioned above:

- for $N_{1}=1$ and $f(\lambda)=\lambda$, the distribution of $\tilde{L}$ corresponds with the distribution of the maximal eigenvalue $\lambda_{1}$.

- The case $N_{1}=N$ reduces to the statistical analysis of the full linear statistics $L$.

We introduce the fraction $\kappa=N_{1} / N$ of charges contributing to the truncated linear statistics and rescale this latter as $s=N^{-\eta} \tilde{L}$, where the exponent $\eta$ controls the large $N$ scaling of $\tilde{L}$ (i.e. $\tilde{L} \sim N^{\eta}$ ) ; the precise value of $\eta$ depends on the ensemble and the function $f$, as we will see later. The main question is here to determine the distribution

$$
\begin{aligned}
& P_{N, \kappa}(s)=N ! \\
& \times \int \mathrm{d} \lambda_{1} \int^{\lambda_{1}} \mathrm{~d} \lambda_{2} \cdots \int^{\lambda_{N-1}} \mathrm{~d} \lambda_{N} P\left(\lambda_{1}, \ldots, \lambda_{N}\right) \delta\left(s-N^{-\eta} \sum_{n=1}^{N_{1}} f\left(\lambda_{n}\right)\right)
\end{aligned}
$$

(we omit to specify the precise domain of integration, which depends on the matrix ensemble).

Although our results are very general and can be applied to many linear statistics or matrix ensembles, we will focus on an example involving Wishart matrices. Such matrix ensembles were introduced by Wishart [37] for the study of empirical covariance in multivariate statistics. They correspond to matrices of the form $Y=X^{\dagger} X$, with $X$ of size $M \times N$ with independent and identically distributed Gaussian entries. It is convenient to introduce the Dyson index $\beta$ corresponding to real $(\beta=1)$, complex $(\beta=2)$ or quaternionic $(\beta=4)$ matrix entries. The eigenvalues of $Y$ are positive, and have the following joint probability density function:

$$
P\left(\lambda_{1}, \ldots, \lambda_{N}\right) \propto \prod_{i<j}\left|\lambda_{j}-\lambda_{j}\right|^{\beta} \prod_{n=1}^{N} \lambda_{n}^{\frac{\beta}{2}(M-N+1)-1} \mathrm{e}^{-\beta \lambda_{n} / 2}, \quad \lambda_{n}>0 .
$$

In this expression, $M \geqslant N$ is an integer, however we will consider below an extension where $M-N+1$ is replaced by a real positive number (Laguerre ensemble of random matrix theory). In Ref. [20], this distribution was shown to arise in the analysis of a model of $1 \mathrm{D}$ interfaces $[38,39]$, which is described in Section 2. 
1.1 Main results

We have analysed the distribution (1.3) in the large $N$ limit with $\kappa=N_{1} / N$ fixed for the Laguerre ensemble, Eq. (1.4) with $M-N=\mathcal{O}(1)$, and $f(\lambda)=\sqrt{\lambda}$. The result can be written under the form ${ }^{2}$

$$
P_{N, \kappa}(s) \underset{N \rightarrow \infty}{\sim} \exp \left\{-\frac{\beta N^{2}}{2} \Phi_{\kappa}(s)\right\},
$$

where the large deviation function has the following limiting behaviours:

$$
\Phi_{\kappa}(s) \simeq \begin{cases}-2 \ln s & \text { as } s \rightarrow 0 \\ \frac{\pi^{2}}{4-c_{0}+c_{0} \ln c_{0} / 4}\left(s-s_{0}(\kappa)\right)^{2} & \text { as } s \rightarrow s_{0}(\kappa) \\ \frac{s^{2}}{\kappa}+\kappa(3 \kappa-4) \ln s & \text { as } s \rightarrow+\infty\end{cases}
$$

$s_{0}(\kappa)$, given parametrically in Eqs. $(3.24,3.25)$, denotes a critical line in the $(\kappa, s)$ plane, as shown in the phase diagram in Fig. 1.2. The constant $c_{0}$ is controlled by $\kappa$, cf. Eq. (3.25) below. A sketch of the distribution is plotted in Fig. 1.1.

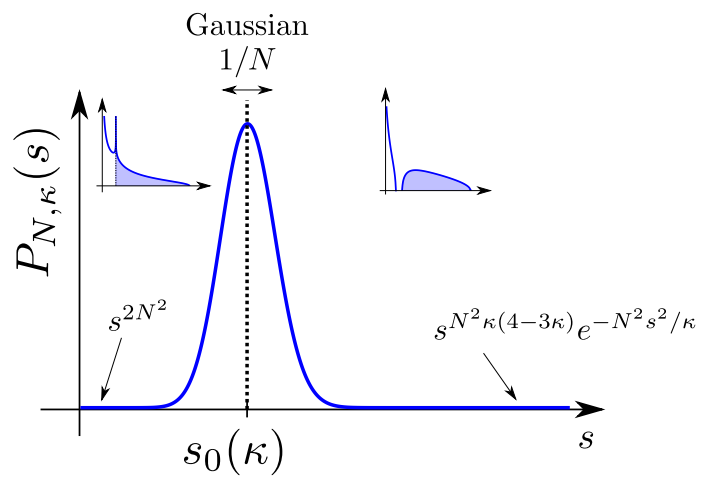

Fig. 1.1 Sketch of the distribution $P_{N, \kappa}(s)$ of the truncated linear statistics.

This specific distribution can be understood from a new universal scenario that we now describe. In the $N \rightarrow \infty$ limit, the multiple integrals (1.3) are dominated by the optimal configuration of charges of the Coulomb gas. The two important parameters that govern the behaviour of the gas are the fraction $\kappa=N_{1} / N$ and the parameter $s$ controlling the constraint $s=$ $N^{-\eta} \sum_{n=1}^{N_{1}} f\left(\lambda_{n}\right)$. For a given $\kappa$, the change of $s$ drives a phase transition

2. In the paper, the expressions for the limiting behaviours of $P_{N, \kappa}(s)$ must be understood more rigorously as $\lim _{N \rightarrow \infty}\left[-2 /\left(\beta N^{2}\right)\right] \ln \left[P_{N, \kappa}(s)\right]=\Phi_{\kappa}(s)$. 
in the Coulomb gas corresponding to a change of the optimal charge density. Phase 1 corresponds to a density supported on two disjoint intervals (see Fig. 1.2). As $s$ approaches the critical value $s_{0}(\kappa)$, the gap between the two intervals shrinks. Exactly at the transition, the density is a smooth function (see Fig. 1.2). If $s$ deviates from $s_{0}(\kappa)$, entering in Phase 2, a logarithmic divergence emerges at the point where the two intervals have merged (see Fig. 1.2). The energy of the Coulomb gas exhibits an essential singularity at the transition $s=s_{0}(\kappa)$, hence the phase transition is of infinite order.

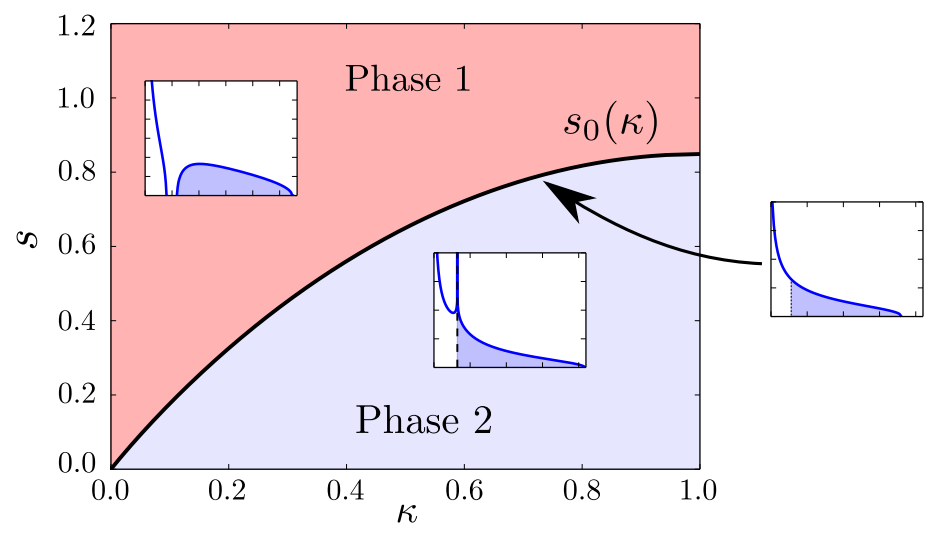

Fig. 1.2 Phase diagram for the Coulomb gas in the $(\kappa, s)$ plane, where $\kappa=N_{1} / N$ and $s=N^{-\eta} \sum_{n=1}^{N_{1}} f\left(\lambda_{n}\right)$ (for the case $f(\lambda)=\sqrt{\lambda}$ in the Laguerre ensemble, with $\eta=3 / 2$ ). Insets show the shape of the corresponding optimal "charge" density profiles (shaded areas correspond to the $N_{1}$ largest eigenvalues). The phase transition occurs on the line $s_{0}(\kappa)$ (thick black line) corresponding to the most probable value of $\sum_{n=1}^{N_{1}} f\left(\lambda_{n}\right)$.

The scenario just described is not restricted to the particular case considered here (Laguerre ensemble with the function $f(\lambda)=\sqrt{\lambda}$ ). We have shown (Section 5 and Appendix A) that this scenario is universal and holds for any monotonous function $f$ and any matrix ensemble, and can be summarised as follows. For fixed $\kappa$, the optimal density of charges undergoes an infinite order phase transition at the critical value $s_{0}(\kappa)$ :

- In the first phase, the density is supported on two disjoint intervals.

- In the second phase, the density exhibits a logarithmic singularity. The general expressions of these densities can be found in Appendix A. Note that depending on the function $f$, other phase transitions might be present (cf. Section 5).

\subsection{Outline of the paper}

The paper is organised as follows: the interface model is described in Section 2. Section 3 presents the Coulomb gas analysis and the determination of 
the large deviation function. The implications of these results for the interface model are discussed in Section 4. Subsection 4.3 describes the outcome of numerical simulations performed to check our analytical results. The paper is closed with some concluding remarks and a brief discussion of another truncated linear statistics within the Jacobi ensemble. Appendix A describes some technical issues and show that the main scenario is robust and not restricted to the specific truncated linear statistics studied in Sections 3 and 4.

\section{The interface model}

Our discussion will be based on a model of $N$ non intersecting $(1+1)$ dimensional elastic fluctuating interfaces. This model was first introduced by de Gennes [40], and later by Fisher [38] in order to study wetting of surfaces or the commensurate/incommensurate transition in deposition of atoms on a surface (for a review, cf. [21]). The interfaces evolve on a substrate of size $L$, which induces a repulsive force. More precisely, each interface is described by its height $h_{n}(x), n=1, \ldots, N$, for $x$ between 0 and $L$ and we assume periodic boundary conditions $h_{n}(L)=h_{n}(0)$. An energy can be associated to the system of $N$ interfaces:

$$
E\left[\left\{h_{n}(x)\right\}\right]=\sum_{n=1}^{N} \mathcal{E}\left[h_{n}(x)\right]
$$

where

$$
\mathcal{E}\left[h_{n}(x)\right]=\int_{0}^{L}\left[\frac{1}{2}\left(\frac{\mathrm{d} h}{\mathrm{~d} x}\right)^{2}+V(h(x))\right] \mathrm{d} x .
$$

$(1 / 2) \int\left(\mathrm{d} h_{n} / \mathrm{d} x\right)^{2}$ is the elastic energy and $V\left(h_{n}\right)$ some external potential. Following the approach of Ref. [20] we choose it in the form

$$
V(h)=\frac{b^{2} h^{2}}{2}+\frac{\alpha(\alpha-1)}{2 h^{2}} \quad \text { with } b>0 \text { and } \alpha>1 .
$$

This potential is made of two terms: a harmonic confining term and a repulsion term from the substrate (supposed at $h=0$ ), which ensures that the interfaces remain in the region $h>0$. This repulsion in $h^{-2}$ is justified by entropic considerations $[20,38]$. The model with purely harmonic confinement was used in order to study vicinal surfaces of cristals (for a review, see Ref. [39]). At thermal equilibrium, a configuration of $N$ interfaces $\left\{h_{n}(x)\right\}_{n=1}^{N}$ can be associated to a Boltzmann weight:

$$
\mathcal{P}\left[\left\{h_{n}(x)\right\}\right] \propto \exp \left\{-\frac{1}{k_{\mathrm{B}} T} E\left[\left\{h_{n}(x)\right\}\right]\right\},
$$

where $T$ is the temperature and $k_{\mathrm{B}}$ the Boltzmann constant. We set $k_{\mathrm{B}} T=1$ for convenience. Moreover we impose that the interfaces do not intersect, hence we can order them: $h_{1}>h_{2}>\cdots>h_{N}$. Our choice of boundary conditions 


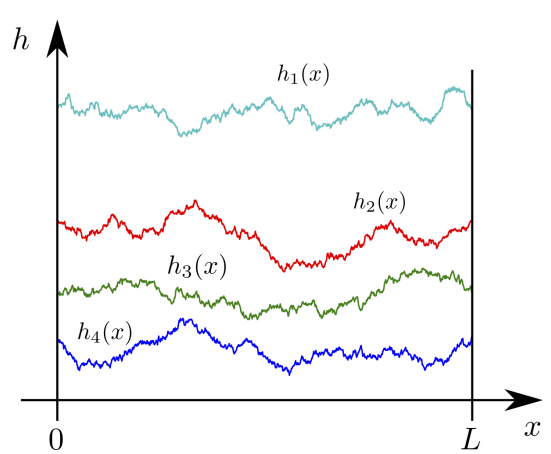

Fig. 2.1 Nonintersecting Brownian interfaces $h_{n}(x)$ with periodic boundary conditions $h_{n}(L)=h_{n}(0)$.

implying translational invariance, the joint distribution of the heights is independent of the position $x$. This distribution was obtained in Ref. [20] by mapping the heights of the interfaces to the positions of free quantum particles trapped in the potential $V(h)$. Since the interfaces cannot intersect, the fictitious particles are fermions. In the limit $L \rightarrow \infty$, their distribution is controlled by the wave function $\Psi_{0}\left(h_{1}, \ldots, h_{N}\right)$ of the many body ground state, which yields [20]:

$$
P_{\text {interfaces }}\left(h_{1}, \ldots, h_{N}\right) \propto \prod_{i<j}\left(h_{i}^{2}-h_{j}^{2}\right)^{2} \prod_{n=1}^{N} h_{n}^{2 \alpha} \mathrm{e}^{-b h_{n}^{2}} .
$$

The Vandermonde determinant $\prod_{i<j}\left(h_{i}^{2}-h_{j}^{2}\right)^{2}$ originates in the fermionic nature of the particles, equivalently, in the condition that the interfaces do not cross. A simple change of variable $\lambda_{n}=b h_{n}^{2}$ allows to relate this distribution to the joint probability density function of eigenvalues for $N \times N$ Wishart matrices (Laguerre ensemble):

$$
P\left(\lambda_{1}, \ldots, \lambda_{N}\right) \propto \prod_{i<j}\left(\lambda_{i}-\lambda_{j}\right)^{2} \prod_{n=1}^{N} \lambda_{n}^{\alpha-1 / 2} \mathrm{e}^{-\lambda_{n}} .
$$

It corresponds to Eq. (1.4) with $\beta=2$ and $M=N+\alpha-1 / 2$. This particular value of $\beta=2$ is not related to the breaking of the time reversal symmetry but can be here understood from the relation between the wave function, given by a Slater determinant, and the probability density $P_{\text {interfaces }}\left(h_{1}, \ldots, h_{N}\right)=\left|\Psi_{0}\left(h_{1}, \ldots, h_{N}\right)\right|^{2}$. The distribution (2.6) will be the starting point of our analysis. We are interested in the distribution of the center of mass of the $N_{1}$ highest interfaces:

$$
G=\frac{1}{N_{1}} \sum_{n=1}^{N_{1}} h_{n} \quad, \quad h_{1}>h_{2}>\cdots>h_{N} .
$$


Denote $\kappa=N_{1} / N$ the fraction of interfaces we consider. This can be rewritten as

$$
G=\frac{1}{N_{1} \sqrt{b}} \sum_{n=1}^{N_{1}} \sqrt{\lambda_{n}} \quad, \quad \lambda_{1}>\lambda_{2}>\cdots>\lambda_{N}
$$

i.e. the function in $(1.2)$ is

$$
f(\lambda)=\sqrt{\lambda}
$$

We will study the limit $N \rightarrow \infty$, with $0<\kappa<1$ fixed, using a Coulomb gas method.

\section{Coulomb gas analysis of the truncated linear statistics}

The Coulomb gas method consists in rewriting the joint distribution (2.6) as a Gibbs measure $\exp \left[-E_{\text {gas }}\right]$, with the energy $E_{\text {gas }}=-\sum_{i \neq j} \ln \left|\lambda_{i}-\lambda_{j}\right|+$ $\sum_{i}\left(\lambda_{i}-(\alpha-1 / 2) \ln \lambda_{i}\right)$. This energy describes a gas of particles on a semiinfinite line, trapped in a confining potential $V(\lambda)=\lambda-(\alpha-1 / 2) \ln \lambda$ and submitted to repulsive logarithmic interaction between each other. In the limit $N \rightarrow \infty$, the interaction energy scales with $N$ as $\sum_{i \neq j} \ln \left|\lambda_{i}-\lambda_{j}\right| \sim N^{2}$, and the confinement energy as $\sum_{i} \lambda_{i} \sim N \lambda$, where $\lambda$ is a typical value taken by the $\lambda_{i}$ 's. Since we expect the distribution of charges to find an equilibrium between confinement and repulsion, the eigenvalues should scale as $\lambda_{i} \sim N$. Hence, we rescale them as

$$
\lambda_{i}=N x_{i}
$$

We can then introduce the empirical density

$$
\rho(x)=\frac{1}{N} \sum_{n=1}^{N} \delta\left(x-x_{n}\right),
$$

and rewrite the measure (2.6) as a functional of this density (we neglect entropic contributions which are of order $N$, compared to the energy of order $\left.N^{2}[28,41]\right)$ :

$$
P\left(\lambda_{1}, \ldots, \lambda_{N}\right) \mathrm{d} \lambda_{1} \cdots \mathrm{d} \lambda_{N} \rightarrow \mathrm{e}^{-N^{2} \mathscr{E}[\rho]} \mathcal{D} \rho
$$

with the energy

$$
\mathscr{E}[\rho]=-\int \rho(x) \rho(y) \ln |x-y| \mathrm{d} x \mathrm{~d} y+\int \rho(x)\left(x-\frac{\alpha-1 / 2}{N} \ln x\right) \mathrm{d} x .
$$

Since we consider only the large $N$ limit, we will drop the $1 / N$ term in the energy, assuming that $\alpha=\mathcal{O}\left(N^{0}\right)$. The rescaled linear statistics (2.8) reads

$$
s=\frac{\kappa \sqrt{b} G}{\sqrt{N}}=\int_{c} \rho(x) \sqrt{x} \mathrm{~d} x
$$


where $c$ is a lower bound ensuring that only the $N_{1}$ largest eigenvalues contribute to the integral, i.e.

$$
\int_{c} \rho(x) \mathrm{d} x=\kappa
$$

Our aim is to compute the distribution of the rescaled center of mass $s$, which can be expressed in terms of path integrals over the density:

$$
\begin{aligned}
& P_{N, \kappa}(s)= \\
& \frac{\int \mathrm{d} c \int \mathcal{D} \rho \mathrm{e}^{-N^{2} \mathscr{E}[\rho]} \delta\left(\int_{c} \mathrm{~d} x \rho(x)-\kappa\right) \delta\left(\int^{c} \mathrm{~d} x \rho(x)-(1-\kappa)\right) \delta\left(\int_{c} \mathrm{~d} x \sqrt{x} \rho(x)-s\right)}{\int \mathrm{d} x \mathrm{~d} c \int \mathcal{D} \rho \mathrm{e}^{-N^{2} \mathscr{E}[\rho]} \delta\left(\int_{c} \mathrm{~d} x \rho(x)-\kappa\right) \delta\left(\int^{c} \mathrm{~d} x \rho(x)-(1-\kappa)\right)}
\end{aligned}
$$

A dual problem was studied in Ref. [33], where the distribution of the number of eigenvalues above a fixed threshold was considered. This corresponds to study the distribution of $\kappa$ with fixed $c$ corresponding to the threshold, while releasing the constraint on $s$. However, here we are interested in the distribution of $s$ with $\kappa$ fixed, and $c$ is now a parameter determined by $\kappa$. The addition of the constraint on $s$ will lead to a completely different phenomenology.

\subsection{Saddle point equations and large deviation function}

When $N \rightarrow \infty$, the path integrals are dominated by the minimum of the energy under the constraints imposed by the Dirac $\delta$-functions. These constraints can be handled by introducing Lagrange multipliers $\mu_{0}^{(1)}, \mu_{0}^{(2)}$ and $\mu_{1}$. Denote the "free energy"

$$
\begin{aligned}
\mathscr{F}\left[\rho ; \mu_{0}^{(1)}, \mu_{0}^{(2)}, \mu_{1}\right] & =\mathscr{E}[\rho]+\mu_{0}^{(1)}\left(\int^{c} \rho(x) \mathrm{d} x-(1-\kappa)\right) \\
& +\mu_{0}^{(2)}\left(\int_{c} \rho(x) \mathrm{d} x-\kappa\right)+\mu_{1}\left(\int_{c} \sqrt{x} \rho(x) \mathrm{d} x-s\right) .
\end{aligned}
$$

The numerator of Eq. (3.7) is dominated by the density of charges $\rho^{\star}(x ; \kappa, s)$ that minimizes $\mathscr{F}$. This density is solution of the saddle point equation

$$
\left.\frac{\delta \mathscr{F}}{\delta \rho(x)}\right|_{\rho^{\star}}=0
$$

implying

$$
2 \int \rho^{\star}(y ; \kappa, s) \ln |x-y| \mathrm{d} y=x+ \begin{cases}\mu_{0}^{(1)} & \text { for } x<c \\ \mu_{0}^{(2)}+\mu_{1} \sqrt{x} & \text { for } x>c\end{cases}
$$

which can be understood as the energy balance for the charge at point $x$ between the confinement and the logarithmic repulsion. The Lagrange multipliers 
$\mu_{0}^{(1)}$ and $\mu_{0}^{(2)}$ correspond to chemical potentials fixing the fraction of eigenvalues respectively below and above $c$. The term with $\mu_{1}$ adds another external potential coming from the constraint on $s$. In order to solve this equation, we first take its derivative and interpret the resulting relation as the equilibrium of the forces exerted on the charge at position $x$ :

$$
2 f \frac{\rho^{\star}(y ; \kappa, s)}{x-y} \mathrm{~d} y=1+ \begin{cases}0 & \text { for } x<c \\ \frac{\mu_{1}}{2 \sqrt{x}} & \text { for } x>c\end{cases}
$$

where the integral is a Cauchy principal value integral. It is convenient to describe the density $\rho^{\star}$ with two functions: $\rho_{2}$ related to the fraction $\kappa$ of eigenvalues under consideration, and $\rho_{1}$ for the others (see Figure 3.1)

$$
\rho_{2}(x)=\frac{1}{N} \sum_{n=1}^{N_{1}} \delta\left(x-x_{n}\right), \quad \rho_{1}(x)=\frac{1}{N} \sum_{n=N_{1}+1}^{N} \delta\left(x-x_{n}\right) .
$$

The confining potential ensures that the eigenvalues remain in a bounded

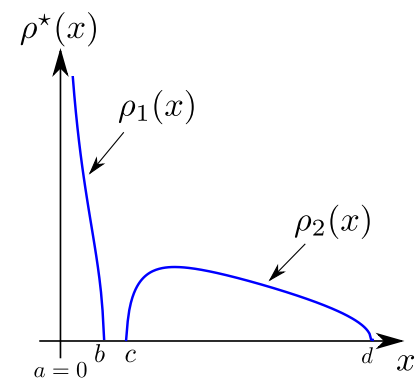

Fig. 3.1 Densities $\rho_{1}$ and $\rho_{2}$ defined by Eq. (3.11) in Phase 1.

region in space, hence the densities $\rho_{1}$ and $\rho_{2}$ will have compact supports. Denote $[a, b]$ the support of $\rho_{1}$, and $[c, d]$ the support of $\rho_{2}$, where $c$ is the boundary introduced above in Eqs. (3.5,3.6), as shown in Figure 3.1. It is possible to have $b=c$, as shown in Fig. 3.2 (left part) and Fig. 5.2.

We rewrite Eq. (3.10) as

$$
\begin{array}{ll}
2 \int_{a}^{b} \frac{\rho_{1}(y)}{x-y} \mathrm{~d} y+2 \int_{c}^{d} \frac{\rho_{2}(y)}{x-y} \mathrm{~d} y=1+\frac{\mu_{1}}{2 \sqrt{x}} & \text { for } x>c \\
2 \int_{a}^{b} \frac{\rho_{1}(y)}{x-y} \mathrm{~d} y+2 \int_{c}^{d} \frac{\rho_{2}(y)}{x-y} \mathrm{~d} y=1 & \text { for } x<c .
\end{array}
$$

Note that, in these two equations, the principal value is only needed when $x$ belongs to the support of the density in the integral. These equations can be solved by a double iteration of a theorem due to Tricomi, as in Ref. [31]. 
This theorem provides an explicit inversion of Cauchy singular equations of the form

$$
f \frac{\rho(y)}{x-y} \mathrm{~d} y=g(x),
$$

under the assumption that the solution has one single support $[a, b]$. This formula reads [42]

$$
\rho(x)=\frac{1}{\pi \sqrt{(x-a)(b-x)}}\left\{A+f_{a}^{b} \frac{\mathrm{d} t}{\pi} \frac{\sqrt{(t-a)(b-t)}}{t-x} g(t)\right\},
$$

where $A$ is a constant. This procedure, detailed in Appendix A, is quite cumbersome, but it is the only one available since the standard method using resolvent technique [26] is even more complicated in this case. The solution of these equations hence give $\rho^{\star}=\rho_{1} \cup \rho_{2}$. The parameters $c$ and $\mu_{1}$ are fixed by the constraints:

$$
\int_{c}^{d} \rho_{2}(x) \mathrm{d} x=\kappa \quad, \quad \int_{c}^{d} \rho_{2}(x) \sqrt{x} \mathrm{~d} x=s .
$$

These two equations give $\mu_{1}$ and $c$ as functions of $s$ and $\kappa$. Let us denote $\mu_{1}^{\star}(\kappa, s)$ and $c^{\star}(\kappa, s)$ the two solutions. The numerator of Eq. (3.7) is dominated by the density $\rho^{\star}$. The optimal density for the denominator can be obtained straightforwardly by releasing the constraint on $s$, which can be done by setting $\mu_{1}^{\star}(\kappa, s)=0$. Solving this equation for $s$ gives $s=s_{0}(\kappa)$, and we will denote the corresponding density $\rho_{0}^{\star}(x)=\rho^{\star}\left(x ; \kappa, s_{0}(\kappa)\right)$. Finally, Eq. (3.7) yields

$$
P_{N, \kappa}(s) \underset{N \rightarrow \infty}{\sim} \exp \left\{-N^{2} \Phi_{\kappa}(s)\right\},
$$

where we have introduced the large deviation function

$$
\Phi_{\kappa}(s)=\mathscr{E}\left[\rho^{\star}(x ; \kappa, s)\right]-\mathscr{E}\left[\rho_{0}^{\star}(x)\right] .
$$

This is the difference of energy between the two optimal configurations of charges dominating the numerator and the denominator of (3.7), respectively. These energies are given by Eq. (3.4). A direct computation of the double integral is quite difficult in practice. However, an important simplification based on a "thermodynamic" identity was introduced in Ref. [12] and discussed in details in Refs. [43, 44]:

$$
\frac{\mathrm{d} \mathscr{E}\left[\rho^{\star}(x ; \kappa, s)\right]}{\mathrm{d} s}=-\mu_{1}^{\star}(\kappa, s) .
$$

Using this relation one can obtain the large deviation function directly by integration of the Lagrange multiplier $\mu_{1}$ :

$$
\Phi_{\kappa}(s)=\int_{s}^{s_{0}} \mu_{1}^{\star}(\kappa, t) \mathrm{d} t
$$

We will make extensive use of this relation in our study of the distribution $P_{N, \kappa}(s)$. To lighten the notations, the dependences on the parameters $\kappa$ and $s$ will be implicit from now on. In particular the optimal density will be simply denoted by $\rho^{\star}(x)$. 
3.2 Optimal density without constraint

In the absence of the constraint $\left(\mu_{1}=0\right)$, the steepest descent equation (3.10) reduces to

$$
2 f \frac{\rho_{0}^{\star}(y)}{x-y} \mathrm{~d} y=1
$$

which can be solved straightforwardly using Tricomi's theorem (3.14,3.15). The density is given by the Marčenko-Pastur distribution [45]:

$$
\rho_{0}^{\star}(x)=\frac{1}{2 \pi} \sqrt{\frac{4-x}{x}} .
$$

The divergence of this density at $x=0$ corresponds to an accumulation of charges, or equivalently of interfaces, near the origin. It has a compact support, meaning that the largest value will typically be $x_{1} \sim 4$. Using the relation $\lambda_{1}=N x_{1}=b h_{1}^{2}$, we deduce that the highest interface will fluctuate around the position

$$
h_{1} \sim 2 \sqrt{N / b}
$$

It is clear from Eq. (3.20) that this distribution is associated to the maximum of the probability, hence to the most probable value of $s$. This value is given by

$$
s_{0}(\kappa)=\int_{c_{0}}^{4} \rho_{0}^{\star}(x) \sqrt{x} \mathrm{~d} x=\frac{\left(4-c_{0}\right)^{3 / 2}}{3 \pi},
$$

where $c_{0}$ is fixed by the fraction of charges under consideration:

$$
\kappa=\int_{c_{0}}^{4} \rho_{0}^{\star}(x) \mathrm{d} x=\frac{2}{\pi} \arccos \frac{\sqrt{c_{0}}}{2}-\frac{\sqrt{c_{0}\left(4-c_{0}\right)}}{2 \pi} .
$$

Solving the second equation for $c_{0}$ and plugging the result into the first gives the most probable value $s_{0}(\kappa)$ taken by the truncated linear statistics, or equivalently, by the center of mass of the highest interfaces. It is the solid line represented in Figure 1.2. In particular, one gets the following asymptotics:

$$
\begin{array}{ll}
\kappa \simeq \frac{\left(4-c_{0}\right)^{3 / 2}}{6 \pi} & \text { as } c_{0} \rightarrow 4, \\
\kappa \simeq 1-\frac{2 \sqrt{c_{0}}}{\pi} & \text { as } c_{0} \rightarrow 0,
\end{array}
$$

from which we deduce:

$$
\begin{array}{ll}
s_{0}(\kappa) \simeq 2 \kappa-\frac{3(3 \pi)^{2 / 3}}{10 \times 2^{1 / 3}} \kappa^{5 / 3} & \text { as } \kappa \rightarrow 0, \\
s_{0}(\kappa) \simeq \frac{8}{3 \pi}-\frac{\pi}{4}(1-\kappa)^{2} & \text { as } \kappa \rightarrow 1 .
\end{array}
$$

We will see later that $s_{0}(\kappa)$ defines a phase transition line (Fig. 1.2). 
3.3 Solution of the saddle point equation

We follow the procedure used in Ref. [31] in a different context. We first solve Eq. (3.13) for $\rho_{1}$ using Tricomi's theorem. This gives a solution in terms of $\rho_{2}$, which can be plugged into Eq. (3.12) to obtain an equation on $\rho_{2}$ only. Then, this equation can be solved using Tricomi's theorem again to get $\rho_{2}$. Finally $\rho_{1}$ can be deduced from the solution of Eq. (3.13). This computation is carried out explicitly in Appendix A in a more general case.

The solution takes a rather simple form, and can be expressed in a compact way in terms of $\rho^{\star}=\rho_{1} \cup \rho_{2}$. The additional term $\mu_{1} \sqrt{x}$ in the potential coming from the constraint pushes the charges either towards or away from the origin depending on the sign of $\mu_{1}$. Therefore we will distinguish these two cases, determined by the sign of $\mu_{1}$.

3.3.1 Phase 1: $\mu_{1}<0$ and $s>s_{0}(\kappa)$ (cf. Fig. 1.2)

We have found the solution of Eqs. $(3.12,3.13)$,

$$
\rho^{\star}(x)=\frac{\mu_{1}}{2 \pi^{2}} \frac{\operatorname{sign}(x-c)}{\sqrt{d-b}} \sqrt{\frac{(c-x)(b-x)}{x(d-x)}} \Pi\left(\frac{d-c}{d-x}, \sqrt{\frac{d-c}{d-b}}\right),
$$

which involves the complete elliptic integral of the third kind, defined by [46]

$$
\Pi(n, k)=\int_{0}^{1} \frac{\mathrm{d} t}{\left(1-n t^{2}\right) \sqrt{\left(1-t^{2}\right)\left(1-k^{2} t^{2}\right)}},
$$

where the integral must be understood in a principal value sense if $n>1$. The fact that the density has a simple analytical form relies on the specific choice (2.9), which is made clear by inspection of $(\mathrm{A} .17, \mathrm{~A} .18)$ : in this case we have $f^{\prime}(\lambda)=1 /(2 \sqrt{\lambda})$, which simplifies the integral in (A.17,A.18) when the boundary is $a=0$. Note that the same formula (3.30) describes the density on the two disjoint intervals $[0, b]$ and $[c, d]$ (see Fig. 3.2, right). To obtain Eq. (3.30), we have imposed that the general form of the density obtained from Tricomi's theorem vanishes at $x=b, c$ and $d\left(\rho^{\star}(d)=0\right.$ is ensured by the elliptic integral). These conditions translate into the following equations:

$$
1+\frac{c-b-d}{4}=\frac{\mu_{1}}{2 \pi}\left[\sqrt{d-b} \mathrm{E}\left(\sqrt{\frac{d-c}{d-b}}\right)-\frac{c-b}{\sqrt{d-b}} \mathrm{~K}\left(\sqrt{\frac{d-c}{d-b}}\right)\right],
$$

coming from the vanishing at $x=b$, and

$$
1+\frac{\mu_{1}}{\pi} \frac{1}{\sqrt{d-b}} \mathrm{~K}\left(\sqrt{\frac{d-c}{d-b}}\right)=0
$$

which arises from the vanishing at $x=d$ (the condition at $x=c$ is already encoded in the previous two expressions). We have denoted $\mathrm{K}$ and $\mathrm{E}$ the complete elliptic integrals of the first and second kind respectively [46]. The last free 
parameters are determined by the two constraints (3.16). The energy balance of the Coulomb gas (3.9) gives some insight to understand why the density splits: the additional potential term coming from the constraint is $\mu_{1} \sqrt{x}$. When $\mu_{1}<0$, this term tends to drive the charges away from the origin. But since this potential affects only the fraction $\kappa$ of the rightmost charges, only these charges are pulled to the right, while the others stay near the origin. Consequently, this gives a value of $s$ larger than the most probable one $s_{0}(\kappa)$. This is coherent with Eq. (3.19), which implies that the energy $\mathscr{E}\left[\rho^{\star}\right]$ associated to this solution increases with $s$, hence a probability decaying as $s$ increases (with $s>s_{0}$ ).
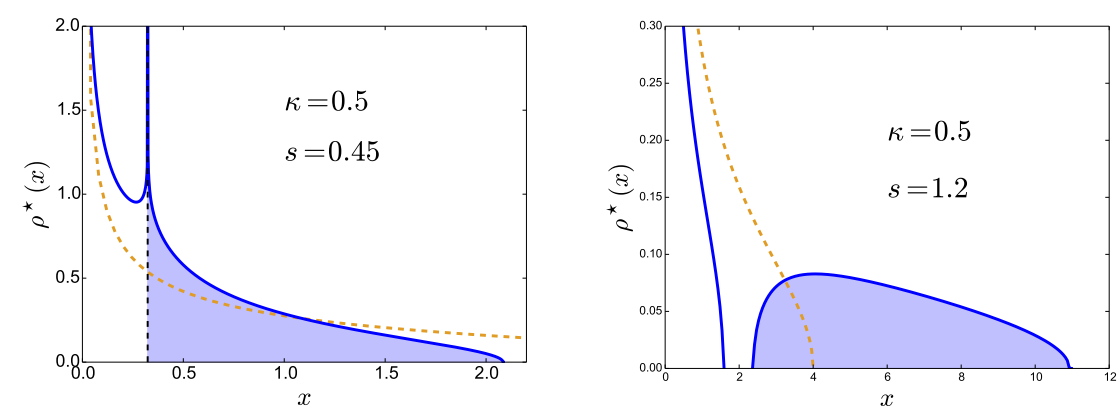

Fig. 3.2 Optimal density $\rho^{\star}$ (plain line), compared to the density in the absence of constraint $\rho_{0}^{\star}$ (dashed). On the left, $\mu_{1}>0$, with $\kappa=0.5$ and $s=0.45$ (Phase 2). On the right, the case $\mu_{1}<0$, with $\kappa=0.5$ and $s=1.2$ (Phase 1). The dashed vertical line delimits the eigenvalues under consideration from the others. At this point, the distribution exhibits a logarithmic singularity.

3.3.2 Phase 2: $\mu_{1}>0$ and $s<s_{0}(\kappa)$ (cf. Fig. 1.2)

Remarkably, the solution of the saddle point equation $(3.12,3.13)$ takes a simple analytical form in this case:

$$
\rho^{\star}(x)=\frac{1}{2 \pi} \sqrt{\frac{d-x}{x}}+\frac{\mu_{1}}{4 \pi^{2} \sqrt{x}} \ln \frac{\sqrt{d-c}+\sqrt{d-x}}{|\sqrt{d-c}-\sqrt{d-x}|} .
$$

The density has one compact support $[0, d]$ (see Fig. 3.2, left), indicating that the two densities $\rho_{1}$ and $\rho_{2}$ merge at $b=c$. To obtain Eq. (3.34), we have imposed that the general solution coming from Tricomi's theorem vanishes at $x=d$. This results in the condition

$$
1-\frac{d}{4}=\frac{\mu_{1}}{2 \pi} \sqrt{d-c}
$$


The remaining free parameters are fixed by the constraints (3.16), which read explicitly:

$$
\begin{aligned}
& \kappa=\frac{d}{2 \pi} \arccos \sqrt{\frac{c}{d}}-\frac{\sqrt{c(d-c)}}{2 \pi}+\frac{\mu_{1}}{2 \pi^{2}}\left(\sqrt{c} \ln \frac{c}{d}+2 \sqrt{d-c} \arccos \sqrt{\frac{c}{d}}\right), \\
& s=\frac{(d-c)^{3 / 2}}{3 \pi}+\frac{\mu_{1}}{2 \pi^{2}}(d-c) .
\end{aligned}
$$

The density exhibits a logarithmic divergence at $x=c$ where $\rho_{1}$ and $\rho_{2}$ merge:

$$
\rho^{\star}(x) \underset{x \rightarrow c}{\simeq}-\frac{\mu_{1}}{4 \pi^{2} \sqrt{c}} \ln |x-c| .
$$

This singularity is clearly visible on Figure 3.2 (left). It is unusual in the framework of the Coulomb gas to obtain such a divergence in the density of eigenvalues. A logarithmic behaviour has already been found at a hard edge in Ref. [20], where the density diverges as $-\ln x / \sqrt{x}$ at the origin. The distribution (3.34) is, to the best of our knowledge, the first example presenting a purely logarithmic divergence in the bulk of a density of eigenvalues. As before, we can interpret this solution in terms of the Coulomb gas: the additional potential $\mu_{1} \sqrt{x}$ felt by the rightmost charges is pushing them towards the origin. But since they must remain to the right of the other charges, the whole density is pushed towards the origin. This phenomenon, caused by the new type of mixed constraints (3.16), is at the origin of the singularity. Although we have discussed this mechanism on the example provided by our model of interfaces, it can be generalized easily to other linear statistics and matrix ensembles (cf. Section 5 and Appendix A).

\subsubsection{Infinite order phase transition}

From the point of view of the Coulomb gas, the parameter $s$ drives a phase transition from a phase where the density is supported on two disjoint intervals $\left(\mu_{1}<0\right)$ to a phase supported by a single interval $\left(\mu_{1}>0\right)$. The transition occurs when $\mu_{1}=0$, which corresponds to the line $s=s_{0}(\kappa)$ in the $(\kappa, s)$ plane, determined by $(3.24,3.25)$ (see Fig. 1.2). On this line, the density is given by the Marčenko-Pastur distribution. One can show that the large deviation function $\Phi_{\kappa}(s)$ and all its derivatives are continuous at this point (Appendix A). However, $\Phi_{\kappa}(s)$ (and also $\mathscr{E}\left[\rho^{\star}(x)\right]$ ) is non analytic. Whereas the function admits a Taylor expansion on one side $\left(s=s_{0}^{-}\right)$, there is an additional essential singularity on the other side of the transition $\left(s=s_{0}^{+}\right)$:

$$
\Phi_{\kappa}\left(s_{0}+\epsilon\right)-\Phi_{\kappa}\left(s_{0}-\epsilon\right)=\mathcal{O}\left(\epsilon \mathrm{e}^{-\gamma / \epsilon}\right),
$$

where

$$
\gamma=\frac{\sqrt{4-c_{0}}}{\pi}\left(4-c_{0}+c_{0} \ln \left(c_{0} / 4\right)\right)
$$


where $c_{0}$ is obtained from (3.25) (a derivation of this result is given in Appendix A.3). Therefore, in the standard terminology of statistical physics, this corresponds to a phase transition of infinite order. Note that, due to the specific choice of the function $f(x)=\sqrt{x}$, a similar singularity was already found in Ref. [20], where the linear statistics (not truncated, i.e. for $\kappa=1$ ) is considered. Let us emphasize that the existence of the essential singularity in the large deviation function of truncated linear statistics $(\kappa<1)$ is a much more universal phenomenon, independent of the choice of $f$, as demonstrated in Appendix A.

\section{Distribution of the center of mass of the highest interfaces}

We first recall the main results obtained in Ref. [20] concerning the distribution of the center of mass of the interfaces, which corresponds to set $N_{1}=N$ (i.e. $\kappa=1$ ). The mean value and the variance were found to be

$$
\langle G\rangle \simeq \frac{8 \sqrt{N}}{3 \pi \sqrt{b}} \quad \text { and } \quad \operatorname{Var}(G) \simeq \frac{2}{\pi^{2} N b}
$$

and the large deviation tails

$$
P_{N, 1}(s) \sim\left\{\begin{array}{l}
s^{2 N^{2}} \text { for } s \rightarrow 0 \\
\mathrm{e}^{-N^{2} s^{2}} \text { for } s \rightarrow \infty
\end{array}\right.
$$

The case $\kappa<1$ is studied by using the solutions $\rho^{\star}$ from the previous section. The large deviation function $\Phi_{\kappa}(s)$ can be easily obtained from the Lagrange multiplier $\mu_{1}$ thanks to Eq. (3.20).

\subsection{Typical values and variance}

The typical value taken by the truncated linear statistics $s$ is the one obtained by relaxing the constraint $\mu_{1}=0$. It corresponds to the value $s_{0}(\kappa)$ defined by Eqs. (3.24,3.25). In the limit $N \rightarrow \infty$, typical and mean value coincide, thus the mean center of mass of the $N_{1}$ highest interfaces is

$$
\langle G\rangle=\frac{\sqrt{N}}{\kappa \sqrt{b}} s_{0}(\kappa) \simeq \frac{1}{\sqrt{b}} \times \begin{cases}2 \sqrt{N}-\frac{3(3 \pi)^{2 / 3}}{10 \times 2^{1 / 3}} \frac{N_{1}^{2 / 3}}{N^{1 / 6}} & \text { for } \kappa=\frac{N_{1}}{N} \rightarrow 0 \\ \frac{8 \sqrt{N}}{3 \pi}\left[1+\frac{N-N_{1}}{N}\right] & \text { for } \kappa \rightarrow 1^{-}\end{cases}
$$

In the limit $\kappa \rightarrow 0$, the center of mass is close to the position of the highest interface, Eq. (3.23). In the limit $\kappa \rightarrow 1$, the leading term corresponds to (4.1), $\langle G\rangle$ being slightly increased by removing the contribution of the lowest interfaces. The variance can be obtained from a Taylor expansion of $\Phi_{\kappa}$ at $s_{0}(\kappa)$. As discussed in Section 3.3.3, the large deviation function has an essential singularity at this point, hence its derivatives are the same on both sides and we 
can restrict the analysis to the case $\mu_{1}>0$ where the expressions are simpler. In the limit $\mu_{1} \rightarrow 0^{+}$, Eqs. $(3.35,3.36,3.37)$ yield

$$
\begin{aligned}
& c=c_{0}+\frac{\mu_{1}}{\pi \sqrt{4-c_{0}}} \ln \frac{c_{0}}{4}+\mathcal{O}\left(\mu_{1}^{2}\right), \\
& d=4-\frac{2 \mu_{1}}{\pi} \sqrt{4-c_{0}}+\mathcal{O}\left(\mu_{1}^{2}\right), \\
& s=s_{0}-\frac{\mu_{1}}{2 \pi^{2}}\left(4-c_{0}+c_{0} \ln \frac{c_{0}}{4}\right)+\mathcal{O}\left(\mu_{1}^{2}\right),
\end{aligned}
$$

The last expression gives $\mu_{1}$ in terms of $s$, hence:

$$
\Phi_{\kappa}(s)=\frac{\pi^{2}}{4-c_{0}+c_{0} \ln c_{0} / 4}\left(s-s_{0}\right)^{2}+\mathcal{O}\left(\left(s-s_{0}\right)^{3}\right) .
$$

The distribution of $s$ exhibits a Gaussian peak around $s_{0}$,

$$
P_{N, \kappa}(s) \underset{s \sim s_{0}}{\sim} \exp \left\{-\frac{N^{2} \pi^{2}}{4-c_{0}+c_{0} \ln c_{0} / 4}\left(s-s_{0}\right)^{2}\right\},
$$

from which we deduce the variance:

$$
\operatorname{Var}(s)=\frac{1}{2 \pi^{2} N^{2}}\left(4-c_{0}+c_{0} \ln \frac{c_{0}}{4}\right)
$$

where $c_{0}$ is determined by the value of $\kappa$ through Eq. (3.25). The scaling of the fluctuations as $1 / N$, i.e. much smaller than $1 / \sqrt{N}$ for independent variables, is a signature of the long range correlations in the Coulomb gas. Coming back to the interface model, we find the corresponding limiting behaviours for the variance of the center of mass:

$$
\operatorname{Var}(G) \simeq \frac{1}{b} \times \begin{cases}\frac{6^{4 / 3}}{16 \pi^{2 / 3} N^{1 / 3} N_{1}^{2 / 3}} & \text { for } \kappa \rightarrow 0 \\ \frac{2}{\pi^{2} N}\left[1+\frac{N-N_{1}}{N}\right] & \text { for } \kappa \rightarrow 1^{-}\end{cases}
$$

The leading term when $\kappa \rightarrow 1$ corresponds to (4.1).

It is quite interesting to compare the limiting behaviours obtained in the regime $N_{1} \ll N(\kappa \rightarrow 0)$ to the extreme statistics for the interfaces positions. The position the uppermost interface $h_{1}$ was shown to be $h_{1}=2 \sqrt{N / b}+\delta h_{1}$, where the typical fluctuations are described by the Tracy-Widom distribution with the scaling $\delta h_{1} \sim N^{-1 / 6}[20]$ (the large deviations of $h_{1}$ are also discussed in this paper). The same scaling $N^{-1 / 6}$ that appears in the distribution of the maximum height distribution, also appears both in the subleading correction term in $\langle G\rangle$ and in the fluctuations. 
4.2 Large deviations

4.2.1 Limit $s \rightarrow \infty$

This corresponds to the case $\mu_{1} \rightarrow-\infty$. In this limit, the fraction $\kappa$ of the rightmost charges are pushed to infinity, while the others remain close to the origin (Fig. 3.2, right). Explicitly, it corresponds to $c, d \rightarrow \infty$, with $\frac{d-c}{d-b} \rightarrow 0$. Combining Eqs. (3.16,3.32,3.33) yields

$$
\begin{aligned}
& b=4(1-\kappa)+8 \kappa(25 \kappa-4) \frac{1}{\mu_{1}^{2}}+\mathcal{O}\left(\mu_{1}^{-3}\right), \\
& c=\frac{\mu_{1}^{2}}{4}+\sqrt{2 \kappa} \mu_{1}+4-\kappa+\mathcal{O}\left(\mu_{1}^{-1}\right), \\
& d=\frac{\mu_{1}^{2}}{4}-\sqrt{2 \kappa} \mu_{1}+4-\kappa+\mathcal{O}\left(\mu_{1}^{-1}\right) .
\end{aligned}
$$

Rescaling $x=c+(d-c) y$, the density $\rho_{2}$ behaves as

$$
(d-c) \rho_{2}(x)=\frac{8 \kappa}{\pi} \sqrt{y(1-y)}\left(1-\frac{3 \sqrt{2 \kappa}}{\mu_{1}}(2 y-1)+\mathcal{O}\left(\mu_{1}^{-2}\right)\right) .
$$

At leading order, the density $\rho_{2}$ is given by the semi-circle law. This is expected since the charges no longer feel the presence of the wall at the origin. Substituting $\rho_{2}(x)$ from Eq. (4.14) in $s=\int \sqrt{x} \rho_{2}(x) \mathrm{d} x$ gives

$$
\mu_{1}=-\frac{2 s}{\kappa}-\frac{\kappa(3 \kappa-1)}{s}+\mathcal{O}\left(s^{-2}\right)
$$

hence after integration, Eq. (3.20),

$$
\Phi_{\kappa}(s)=\frac{s^{2}}{\kappa}+\kappa(3 \kappa-4) \ln s+\mathcal{O}(1)
$$

corresponding to the behaviour

$$
P_{N, \kappa}(s) \underset{s \rightarrow \infty}{\sim} s^{N^{2} \kappa(4-3 \kappa)} \mathrm{e}^{-N^{2} s^{2} / \kappa} .
$$

When $\kappa=1$, the dominant exponential term corresponds to (4.2). In addition to the generalization to $\kappa<1$, we also provide the next term, which gives the power law in the distribution of $s$. The exponential term can be obtained easily by a heuristic argument based on the Coulomb gas picture: for large $s$, the energy is dominated by the potential energy of the charges pushed to infinity. The typical value of the position of these charges is given by $s \sim \kappa \sqrt{x_{\text {typ }}}$, corresponding to $x_{\text {typ }} \sim s^{2} / \kappa^{2}$. The energy of these charges is estimated as $\mathscr{E}\left[\rho^{\star}(x)\right] \sim \int_{c} \rho^{\star} V \sim \kappa V\left(x_{\mathrm{typ}}\right) \sim s^{2} / \kappa$. QED. 
4.2.2 Limit $s \rightarrow 0$

In this case, all the charges are pushed towards the origin, meaning $0<$ $c<d \rightarrow 0$. In this limit, Eqs. (3.35,3.37) yield $\mu_{1} \simeq 2 \pi / \sqrt{d-c}$ and $s \simeq$ $\mu_{1}(d-c) /\left(2 \pi^{2}\right)$. This gives the behaviour of the Lagrange multiplier $\mu_{1} \simeq 2 / s$. The large deviation function is again deduced by integration with respect to $s$, Eq. (3.20):

$$
\Phi_{\kappa}(s) \simeq-2 \ln s \quad \text { as } s \rightarrow 0 .
$$

Equivalently, the distribution of $s$ behaves as

$$
P_{N, \kappa}(s) \underset{s \rightarrow 0}{\sim} s^{2 N^{2}}
$$

Remarkably, the left tail of the distribution does not depend on the fraction $\kappa$ of eigenvalues considered, and coincides precisely with (4.2). This can be understood as follows: in the limit $s \rightarrow 0$ all the charges are pushed towards the origin, so the energy of the Coulomb gas is dominated by the interaction term describing logarithmic repulsion among charges. The typical distance between two charges is $\delta x=x_{\text {typ }} / N$, hence $\mathscr{E}\left[\rho^{\star}(x)\right] \sim-\ln \delta x \sim-2 \ln s$.

\subsection{Numerics}

We have also performed Monte Carlo simulations of the Coulomb gas. One starts from a trial distribution of $N$ charges that fulfills the constraint (1.2). Then, pairs of charges are randomly moved in a way that preserves the constraint (1.2), with acception or rejection rule according to the Metropolis algorithm. The density eventually relaxes towards the optimal density with minimum energy. All the simulations were performed for $N=2000$ charges.
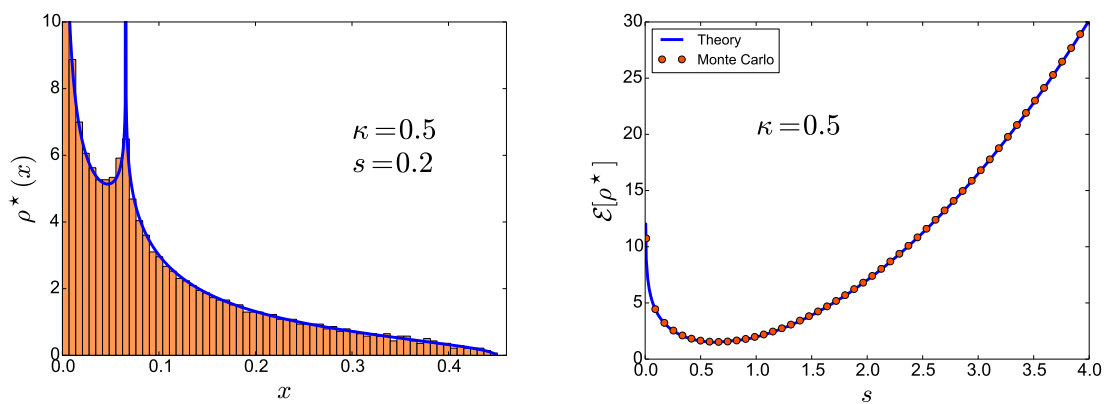

Fig. 4.1 Monte Carlo simulations for the Coulomb gas, with $N=2000$ charges and $\kappa=0.5$. Left: histogram of the density obtained numerically for $s=0.2$ compared to the plot of Eq. (3.34) (no fit). The logarithmic divergence is clearly visible. Right: energy of the Coulomb gas (large deviation function up to a constant) obtained from the simulation compared to numerical integration of Eq. (3.4) using the densities (3.30,3.34). 
The density obtained numerically matches perfectly our computation (see the left part of Fig. 4.1). In particular, the logarithmic divergence is clearly visible. The large deviation function $\Phi_{\kappa}(s)$ is also in perfect agreement with our results (Fig. 4.1, right). We stress that the comparison of the numerical and analytical results does not involve any adjustable parameter.

\section{Conclusion}

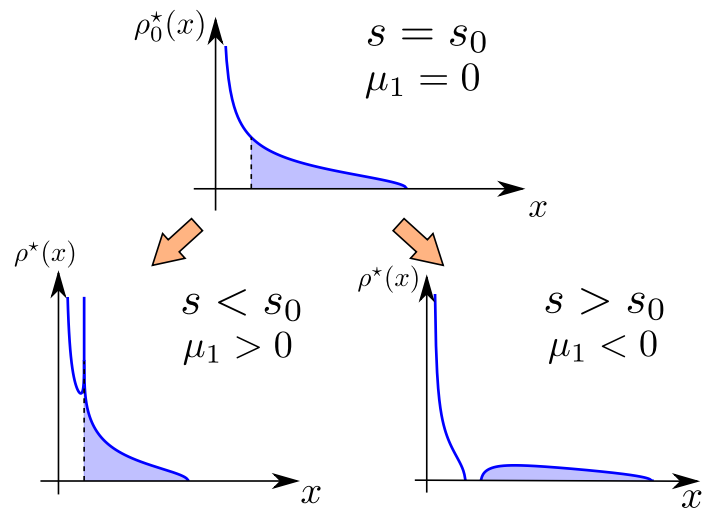

Fig. 5.1 Sketch of the mechanism described in this paper. The optimal density $\rho^{\star}$ of the gas undergoes a phase transition driven by the constraint imposed by (1.2). $\rho_{0}^{\star}$ is the density obtained when the constraint is released.

In this paper we have studied an example of truncated linear statistics of top eigenvalues $\tilde{L}=\sum_{n=1}^{N_{1}} f\left(\lambda_{n}\right)$ within the Laguerre ensemble of random matrices. In the large $N$ limit, by using the Coulomb gas technique, the problem has been recast as an optimization problem where we have searched for the most probable charge configuration consistent with the constraint that $s=N^{-\eta} \sum_{n=1}^{N_{1}} f\left(\lambda_{n}\right)$ is fixed ; $\eta$ is an exponent ensuring that $s$ scales as $\mathcal{O}\left(N^{0}\right)$ when $N \rightarrow \infty$ (e.g. $\eta=3 / 2$ for $f(\lambda)=\sqrt{\lambda}$ in the Laguerre ensemble). When the constraint is removed, the optimal charge density $\rho_{0}^{\star}$ is the Marčenko-Pastur density, which thus provides the typical (most probable) value of the truncated linear statistics:

$$
s_{0}(\kappa)=\int_{c_{0}} \rho_{0}^{\star}(x) f(x) \mathrm{d} x, \quad \text { where } \quad \kappa=\int_{c_{0}} \rho_{0}^{\star}(x) \mathrm{d} x .
$$

These two equations define a line $s=s_{0}(\kappa)$ in the $(\kappa, s)$ plane, which was shown to correspond to a phase transition line of infinite order (essential singularity in the energy). This line separates two phases characterized by different density profiles (Fig. 1.2): on one side the density is supported on two disconnected intervals and on the other side the density has a compact support. In this second case, the density presents a logarithmic divergence inside the bulk (the 
main mechanism is sketched in Fig. 5.1). Although these new results were obtained within the Laguerre ensemble and for the specific choice of truncated linear statistics $\tilde{L}=\sum_{n=1}^{N_{1}} \sqrt{\lambda_{n}}$ which has arisen from a model of Brownian interfaces, they are quite universal. We have demonstrated in Appendix A that the mechanism is independent of the choice of the function $f$, provided it is monotonous. It is also independent of the matrix ensemble. The case where the function $f$ is non monotonous is however still an open question (this is for example the case for the shot noise of chaotic cavity [10]).

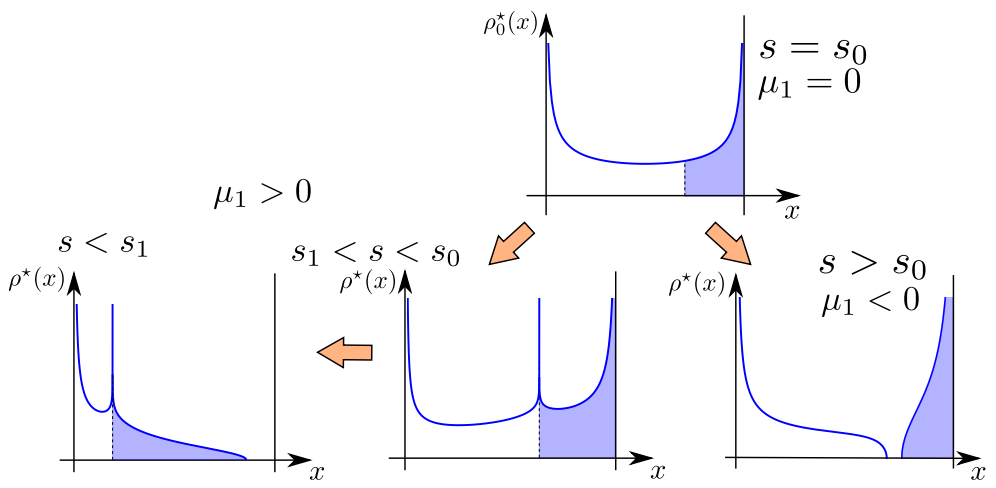

Fig. 5.2 Sketch of the evolution of the density of eigenvalues in the Jacobi ensemble for different values of the linear statistics $s$ for $f(\lambda)=\lambda$. For $s$ in the neighbourhood of the typical value $s_{0}(\kappa)$, the gas follows the same scenario as for the Laguerre ensebmle. There is however an additional third order transition at $s=s_{1}<s_{0}$.

We stress that, depending on the function $f$ and the matrix ensemble, the whole picture may be richer than in the case considered in the paper. We have also studied the truncated linear statistics $\tilde{L}=\sum_{n=1}^{N_{1}} \lambda_{n}$ within the Jacobi ensemble, corresponding to the joint probability density function

$$
P\left(\lambda_{1}, \ldots, \lambda_{N}\right) \propto \prod_{i<j}\left|\lambda_{i}-\lambda_{j}\right|^{\beta} \prod_{n=1}^{N} \lambda_{n}^{\frac{\beta}{2}-1}, \quad 0<\lambda_{n}<1
$$

Since the eigenvalues are bounded, $\tilde{L}$ now remains between 0 and $N_{1}$ and therefore $s=\tilde{L} / N \in[0, \kappa]$. The forbidden region $s>\kappa$ is dashed in Fig. 5.3. The scenario introduced in the paper (Fig. 5.1) is only part of the full scenario as the constraint drives a second phase transition from a hard to a soft edge, as sketched in Fig. 5.2 (this second transition is of third order [10]). As a consequence the whole phase diagram is richer, as shown in Fig. 5.3.

We now make few remarks concerning the two limits $\kappa \rightarrow 0$ and $\kappa \rightarrow 1$. In the case $N_{1}=1$ with $f(\lambda)=\lambda$, the study of the truncated linear statistic distribution is exactly mapped onto the study of the largest eigenvalue, a problem which has been widely discussed in the literature [19,20, 27, 28, 47-49]. However our Coulomb gas analysis in Section 3 cannot be used to consider the 


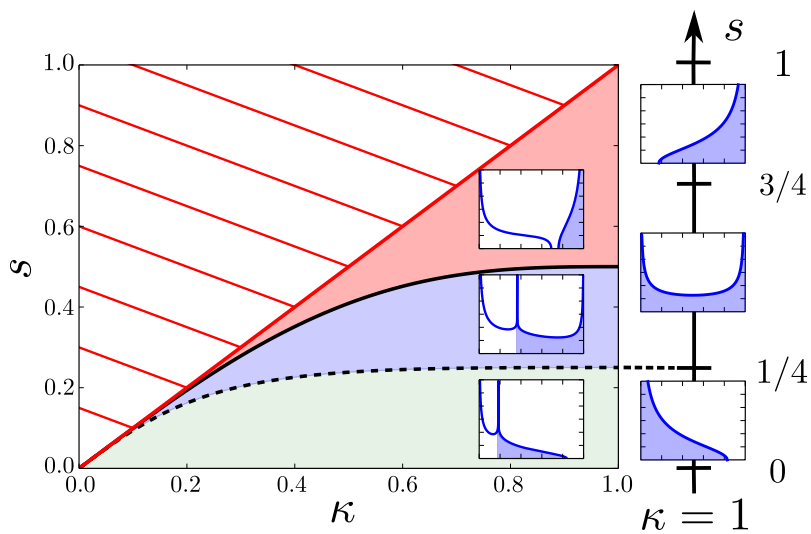

Fig. 5.3 Phase diagram in the Jacobi ensemble for the linear statistics $f(x)=x$. Since the eigenvalues are bounded, $s \in[0, \kappa]$ (the dashed region is the forbidden area). The black line corresponds to $s=s_{0}(\kappa)$, where the infinite order phase transition occurs. The dashed line, say $s=s_{1}(\kappa)$, corresponds to the phase transition from a hard edge to a soft edge, which is third order. The right of the figure shows the phase diagram for $\kappa=1$ with the two third order phase transitions at $s=1 / 4$ and $s=3 / 4$. While the dashed line continues all the way to $\kappa=1$ (with $\left.s_{1}(\kappa \rightarrow 1)=1 / 4\right)$, the solid line stops before $\kappa=1\left(\right.$ with $\left.s_{0}\left(\kappa \rightarrow 1^{-}\right)=1 / 2\right)$.

case $\kappa=1 / N$ as we have studied the thermodynamic limit $N \rightarrow \infty$ with $\kappa$ fixed and have only obtained the leading order contribution to the energy of the gas.

In Section 4, the study of the Laguerre ensemble with $f(\lambda)=\sqrt{\lambda}$ has shown that the large deviation function for the truncated linear statistics $(\kappa<1)$ continuously goes towards the one obtained in Ref. [20] $(\kappa=1)$. We emphasize that this is not a general feature. For $0<\kappa<1$, the (infinite order) phase transition takes place at the typical value $s=s_{0}(\kappa)$. On the other hand, for $\kappa=1$ (full linear statistics), the typical value $s_{0}(1)$ does not correspond in general to a phase boundary, ${ }^{3}$

The singular nature of the two limits is clearly related to non commutation of the limits $\lim _{\kappa \rightarrow 1}\left(\right.$ or $\left.\lim _{\kappa \rightarrow 0}\right)$ and $\lim _{N \rightarrow \infty}$. In particular it would be interesting to study precisely the boundary of the phase diagram for $\kappa \rightarrow 1$ beyond the thermodynamic limit (the region of the phase diagram of Fig. 5.3 where $\kappa \sim 1$ ). Such a general analysis would certainly be interesting as it is known that the Coulomb gas may present very diverse behaviours depending on the ensemble and the function $f$ (cf. Table 5.1).

Much effort has been devoted to analyse the eigenvalue correlations in random matrices. Their universal character was underlined and has led to different

3. The linear statistics $L=\sum_{n=1}^{N} \sqrt{\lambda_{n}}$ in the Laguerre ensemble is quite specific: the typical value corresponds to a phase transition and the density presents an unusual additional logarithmic behaviour at the hard edge, $\rho(x) \sim-\ln x / \sqrt{x}$ as $x \rightarrow 0$ [20]. hence the limit $\kappa \rightarrow 1$ is in general also a singular limit. For example, for $L=\sum_{n=1}^{N} \lambda_{n}$ within the Jacobi ensemble, we clearly see in Fig. 5.3 that the line of infinite order phase transition terminates at $s_{0}(1)=1 / 2$, which is below the third order phase transition occuring at $s=3 / 4$ when $\kappa=1$. 


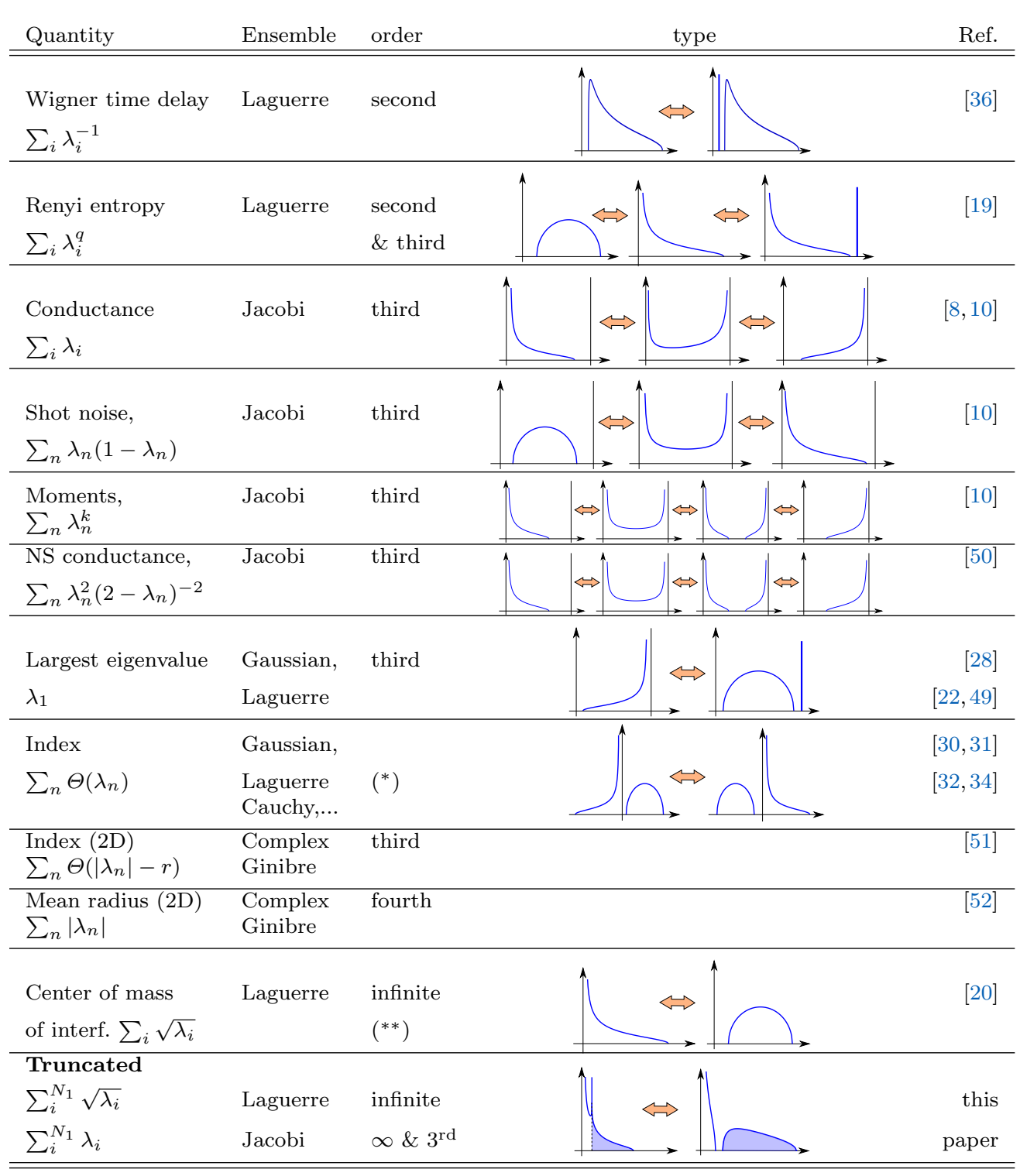

Table 5.1 List of different phase transitions observed in the Coulomb gas. (*) : the energy has a logarithmic correction at the typical value. (**) : the density presents a logarithmic correction at the hard edge $\rho(x) \sim-\ln x / \sqrt{x}$ for $x \rightarrow 0$.

types of correlations: sine kernel in the bulk of the density, Airy kernel in the vicinity of a soft edge and Bessel kernel in the vicinity of a hard edge [53,54]. The existence of a new type of behaviour in the density (logarithmic divergence inside the bulk) thus call for the study of the corresponding eigenvalue correlations. 
Although the physical motivation for the analysis of truncated linear statistics presented here came from the model of Brownian interfaces, we expect that it also applies to other physical situations. Cold atoms could be a possible field of application: it is known that the eigenvalues $\lambda_{i}$ of a matrix from the Gaussian Unitary ensemble correspond to the positions of 1D spinless free fermions in a harmonic well [34]. Hence, many physical quantities can be expressed as linear statistics of the $\lambda_{i}$ 's. Any restriction of these quantities to a given number $N_{1}$ of the rightmost fermions can therefore be treated as a truncated of the largest eigenvalues $\lambda_{1}, \ldots, \lambda_{N_{1}}$, which might be of experimental interest with the recent progresses in measurement of the atom density [55-57].

\section{Acknowledgements}

We are thankful to Gernot Akemann and Grégory Schehr for interesting remarks.

\section{A Solution of the saddle point equation (general analysis within the Laguerre ensemble)}

We study here a more general case than the one discussed in the body of the paper. First, we consider the Laguerre ensemble of random matrix theory corresponding to the joint distribution of eigenvalues:

$$
P\left(x_{1}, \ldots, x_{N}\right) \propto \prod_{i<j}\left|x_{i}-x_{j}\right|^{\beta} \prod_{n=1}^{N} x_{n}^{\beta N \nu / 2} \mathrm{e}^{-\beta N x_{n} / 2}, \quad x_{n}>0,
$$

where $\beta$ is the Dyson index (the eigenvalues have already been rescaled by a factor $N$ so they remain of order one in the limit $N \rightarrow \infty)$. The exponent $\nu \geqslant 0$ is now supposed of order $\mathcal{O}\left(N^{0}\right)$ (in the text, we considered $\nu=0$ ). Note that the computation can be carried out in a similar way for other matrix ensembles. Second, the function $f$ entering in the truncated linear statistics

$$
s=\frac{1}{N} \sum_{n=1}^{N_{1}} f\left(x_{n}\right), \quad x_{1}>x_{2}>\cdots>x_{N}
$$

is now an arbitrary monotonous function (the case $f(\lambda)=\sqrt{\lambda}$ was considered in the text.

Again, we study the limit $N \rightarrow \infty$ with $\kappa=N_{1} / N$ fixed. Using the same notations as in Section 3, the saddle point equation (3.9) becomes:

$$
\begin{array}{ll}
2 \int \frac{\rho_{2}(y)}{x-y} \mathrm{~d} y+2 \int \frac{\rho_{1}(y)}{x-y} \mathrm{~d} y=1-\frac{\nu}{x}+\mu_{1} f^{\prime}(x) & \text { for } x>c, \\
2 \int \frac{\rho_{2}(y)}{x-y} \mathrm{~d} y+2 \int \frac{\rho_{1}(y)}{x-y} \mathrm{~d} y=1-\frac{\nu}{x} & \text { for } x<c,
\end{array}
$$

Assuming the support of $\rho_{1}$ is $[a, b]$ and the support of $\rho_{2}$ is $[c, d]$, the second equation gives $\rho_{1}$ in terms of $\rho_{2}$ using Tricomi's theorem (3.14,3.15):

$$
\rho_{1}(x)=\frac{1}{\pi \sqrt{(x-a)(b-x)}}\left\{A+f_{a}^{b} \frac{\mathrm{d} t}{\pi} \frac{\sqrt{(t-a)(b-t)}}{t-x}\left[\frac{1}{2}\left(1-\frac{\nu}{x}\right)-\int_{c}^{d} \frac{\rho_{2}(y)}{t-y} \mathrm{~d} y\right]\right\} .
$$


The constant $A$ is the normalisation of the density $\rho_{1}$,

$$
\int_{a}^{b} \rho_{1}(x) \mathrm{d} x=A
$$

The principal value integral can be computed by making use of the following relations

$$
\begin{gathered}
f_{a}^{b} \frac{\mathrm{d} t}{\pi} \frac{\sqrt{(t-a)(b-t)}}{t-x}=\frac{a+b}{2}-x, \\
f_{a}^{b} \frac{\mathrm{d} t}{\pi} \frac{\sqrt{(t-a)(b-t)}}{t-x} \frac{1}{t}=-1+\frac{\sqrt{a b}}{x},
\end{gathered}
$$

which hold for $0 \leqslant a<x<b$. The remaining term can be evaluated by permuting the integrals:

$$
f_{a}^{b} \frac{\mathrm{d} t}{\pi} \frac{\sqrt{(t-a)(b-t)}}{t-x} \int_{c}^{d} \frac{\rho_{2}(y)}{t-y} \mathrm{~d} y=\int_{c}^{d} \mathrm{~d} y \rho_{2}(y) f_{a}^{b} \frac{\mathrm{d} t}{\pi} \frac{\sqrt{(t-a)(b-t)}}{t-x} \frac{1}{t-y} .
$$

Using now

$$
f_{a}^{b} \frac{\mathrm{d} t}{\pi} \frac{\sqrt{(t-a)(b-t)}}{t-x} \frac{1}{t-y}=-1+\frac{\sqrt{(y-a)(y-b)}}{y-x}, \quad \text { for } \quad y>b,
$$

we obtain:

$$
f_{a}^{b} \frac{\mathrm{d} t}{\pi} \frac{\sqrt{(t-a)(b-t)}}{t-x} \int_{c}^{d} \frac{\rho_{2}(y)}{t-y} \mathrm{~d} y=-\int_{c}^{d} \rho_{2}(y) \mathrm{d} y+\int_{c}^{d} \rho_{2}(y) \frac{\sqrt{(y-a)(y-b)}}{y-x} \mathrm{~d} y .
$$

Using Eqs. (A.6,A.7,A.8,A.11), we can rewrite Eq. (A.5) as

$$
\begin{aligned}
\rho_{1}(x)=\frac{1}{\pi \sqrt{(x-a)(b-x)}} & \left\{\int_{a}^{b} \rho_{1}(x) \mathrm{d} x+\frac{1}{2}\left[\frac{a+b}{2}-x-\nu\left(\frac{\sqrt{a b}}{x}-1\right)\right]\right. \\
& \left.+\int_{c}^{d} \rho_{2}(y) \mathrm{d} y-\int_{c}^{d} \rho_{2}(y) \frac{\sqrt{(y-a)(y-b)}}{y-x} \mathrm{~d} y\right\} .
\end{aligned}
$$

Since $\int_{a}^{b} \rho_{1}(x) \mathrm{d} x+\int_{c}^{d} \rho_{2}(x) \mathrm{d} x=1$, this expression reduces to

$$
\begin{aligned}
\rho_{1}(x)=\frac{1}{\pi \sqrt{(x-a)(b-x)}\left\{1+\frac{1}{2}\right.} & {\left[\frac{a+b}{2}-x-\nu\left(\frac{\sqrt{a b}}{x}-1\right)\right] } \\
& \left.-\int_{c}^{d} \rho_{2}(y) \frac{\sqrt{(y-a)(y-b)}}{y-x} \mathrm{~d} y\right\} .
\end{aligned}
$$

A divergence $\rho(x) \sim(b-x)^{-1 / 2}$ at the edge of a support is usually caused by a hard wall, which is absent here at $x=b$. Therefore we expect that the expression within the brackets vanishes at $x=b$. Imposing this condition leads to the more compact form:

$$
\rho_{1}(x)=\frac{1}{2 \pi} \sqrt{\frac{b-x}{x-a}}\left\{1-\frac{\nu}{x} \sqrt{\frac{a}{b}}+2 \int_{c}^{d} \sqrt{\frac{y-a}{y-b}} \frac{\rho_{2}(y)}{y-x} \mathrm{~d} y\right\} .
$$

Plugging this expression into (A.3) yields an equation on $\rho_{2}$ only:

$$
2 f \frac{\rho_{2}(t)}{x-t} \sqrt{\frac{t-a}{t-b}} \mathrm{~d} t=1-\frac{\nu}{x} \sqrt{\frac{a}{b}}+\mu_{1} f^{\prime}(x) \sqrt{\frac{x-a}{x-b}},
$$

which can be solved by applying Tricomi's theorem $(3.14,3.15)$ once more. The additional potential $\mu_{1} f$ coming from the constraint either confines or pushed the charges away from the origin, hence the type of solution will depend on the sign of $\mu_{1} f^{\prime}$. In the body of the paper we considered the case $f(x)=\sqrt{x}$. Therefore, it was only required to discuss the sign of $\mu_{1}$. But here the sign of $f^{\prime}$ is important to determine whether Phase 1 corresponds to $\mu_{1}<0$ (as before) or $\mu_{1}>0$, and similarly for Phase 2 . 
A.1 Phase 1: $\mu_{1} f^{\prime}<0$

This corresponds to the situation in which the largest eigenvalues are pushed to the right, giving a density supported on two disjoint intervals (like Phase 1 in Fig. 1.2). Imposing that the densities vanish on the edges of their supports, we obtain:

$$
\begin{aligned}
& \rho_{1}(x)=\frac{1}{2 \pi} \sqrt{\frac{(b-x)(d-x)}{(x-a)(c-x)}}\left\{1-\frac{\nu}{x} \sqrt{\frac{a c}{b d}}+\mu_{1} \int_{c}^{d} \frac{\mathrm{d} t}{\pi} \frac{f^{\prime}(t)}{t-x} \sqrt{\frac{(t-a)(t-c)}{(t-b)(d-t)}}\right\}, \\
& \rho_{2}(x)=\frac{1}{2 \pi} \sqrt{\frac{(x-b)(d-x)}{(x-a)(x-c)}}\left\{1-\frac{\nu}{x} \sqrt{\frac{a c}{b d}}+\mu_{1} f_{c}^{d} \frac{\mathrm{d} t}{\pi} \frac{f^{\prime}(t)}{t-x} \sqrt{\frac{(t-a)(t-c)}{(t-b)(d-t)}}\right\},
\end{aligned}
$$

along with the conditions:

$$
1-\nu \sqrt{\frac{a}{b c d}}+\mu_{1} \int_{c}^{d} \frac{\mathrm{d} t}{\pi} \sqrt{\frac{t-a}{(t-b)(t-c)(d-t)}} f^{\prime}(t)=0,
$$

deduced by imposing $\rho_{2}(c)=0$, and

$$
1+\frac{\nu}{2}+\frac{a+c-b-d}{4}-\frac{\nu}{2} \sqrt{\frac{a c}{b d}}-\frac{\mu_{1}}{2} \int_{c}^{d} \frac{\mathrm{d} t}{\pi} \sqrt{\frac{(t-a)(t-c)}{(t-b)(d-t)}} f^{\prime}(t)=0,
$$

obtained in the derivation of (A.17,A.18) when imposing that the solution of (A.16) vanishes at $x=d$. One should now distinguish two cases:

- $\nu>0$ : in this case $a>0$ and the condition $\rho_{1}(a)=0$ is explicitely

$$
1-\nu \sqrt{\frac{c}{a b d}}+\mu_{1} \int_{c}^{d} \frac{\mathrm{d} t}{\pi} \sqrt{\frac{t-c}{(t-a)(t-b)(d-t)}} f^{\prime}(t)=0 .
$$

- $\nu=0$ : the boundary of the support is the origin, $a=0$.

The parameters $c$ and $\mu_{1}$ are fixed by the constraints

$$
\int \rho_{2}(x) \mathrm{d} x=\kappa \quad \text { and } \quad \int \rho_{2}(x) f(x) \mathrm{d} x=s .
$$

A.2 Phase 2: $\mu_{1} f^{\prime}>0$

In this case, the eigenvalues are pushed towards the origin, and the two densities merge: $b=c$ (like Phase 2 in Fig. 1.2). Similarly, we obtain:

$$
\begin{aligned}
& \rho_{1}(x)=\frac{1}{2 \pi} \sqrt{\frac{d-x}{x-a}}\left\{1-\frac{\nu}{x} \sqrt{\frac{a}{d}}+\mu_{1} \int_{c}^{d} \frac{\mathrm{d} t}{\pi} \sqrt{\frac{t-a}{d-t}} \frac{f^{\prime}(t)}{t-x}\right\}, \\
& \rho_{2}(x)=\frac{1}{2 \pi} \sqrt{\frac{d-x}{x-a}}\left\{1-\frac{\nu}{x} \sqrt{\frac{a}{d}}+\mu_{1} \int_{c}^{d} \frac{\mathrm{d} t}{\pi} \sqrt{\frac{t-a}{d-t}} \frac{f^{\prime}(t)}{t-x}\right\} .
\end{aligned}
$$

To obtain these expressions, we have imposed that the general solution of (A.16) vanishes for $x=d$, which gives the condition

$$
1+\frac{\nu}{2}+\frac{a-d}{4}-\frac{\nu}{2} \sqrt{\frac{a}{d}}=\frac{\mu_{1}}{2} \int_{c}^{d} \frac{\mathrm{d} t}{\pi} \sqrt{\frac{t-a}{d-t}} f^{\prime}(t) .
$$

- $\nu>0$ : in this case $a>0$ and we impose $\rho_{1}(a)=0$,

$$
1-\frac{\nu}{\sqrt{a d}}+\mu_{1} \int_{c}^{d} \frac{\mathrm{d} t}{\pi} \frac{f^{\prime}(t)}{\sqrt{(t-a)(d-t)}}=0 .
$$


- $\nu=0$ : the support's boundary is $a=0$.

Again, the parameters $\mu_{1}$ and $c$ are fixed by the constraints (A.22). In addition, when $x \rightarrow c^{-}$,

$$
\int_{c}^{d} \frac{\mathrm{d} t}{\pi} \sqrt{\frac{t-a}{d-t}} \frac{f^{\prime}(t)}{t-x} \simeq-\frac{f^{\prime}(c)}{\pi} \sqrt{\frac{c-a}{d-c}} \ln |x-c| .
$$

This equation is also valid if one considers the same integral with $x>c$, provided the integral is a Cauchy principal value integral. This shows that the density of eigenvalues exhibits a logarithmic divergence at $x=c$ :

$$
\rho^{\star}(x) \simeq-\frac{\mu_{1}}{2} f^{\prime}(c) \ln |x-c|
$$

This analysis demonstrates that the presence of the logarithmic singularity is not specific to the model studied in the body of the paper, but a direct consequence of the restriction of the linear statistics $s$ to the largest eigenvalues.

\section{A.3 Phase transition of infinite order}

We again interpret the transition between these two types of densities as a phase transition for the Coulomb gas. The transition occurs when $\mu_{1}=0$, and the corresponding density of eigenvalues is given by the Marčenko-Pastur law:

$$
\rho_{0}^{\star}(x)=\frac{\sqrt{\left(x-x_{-}\right)\left(x_{+}-x\right)}}{2 \pi x} \quad \text { where } x_{ \pm}=2+\nu \pm 2 \sqrt{1+\nu} .
$$

The value of the parameter $c$ is then given by

$$
\kappa=\int_{c}^{x+} \rho_{0}^{\star}(x) \mathrm{d} x,
$$

and the corresponding value of the truncated linear statistics is

$$
s=s_{0}(\kappa)=\int_{c}^{x_{+}} \rho_{0}^{\star}(x) f(x) \mathrm{d} x .
$$

Let us study the case $\nu>0$. In the first phase, the limit $\mu_{1} f^{\prime} \rightarrow 0^{-}$corresponds to the case where the two supports merge $(b \rightarrow c)$. In this limit, Eqs. (A.19,A.20,A.21,A.22) reduce to:

$$
\begin{aligned}
& \mu_{1} \simeq \frac{\alpha}{\ln (c-b)} \quad \text { where } \alpha=\frac{\pi}{f^{\prime}(c)} \sqrt{\frac{d-c}{c-a}}\left(1-\frac{\nu}{c} \sqrt{\frac{a}{d}}\right), \\
& 1+\frac{\nu}{2}+\frac{a-d}{4}-\frac{\nu}{2} \sqrt{\frac{a}{d}}=\frac{\mu_{1}}{2} \int_{c}^{d} \frac{\mathrm{d} t}{\pi} \sqrt{\frac{t-a}{d-t}} f^{\prime}(t)+\mathcal{O}\left(\mathrm{e}^{\alpha / \mu_{1}}\right), \\
& 1-\frac{\nu}{\sqrt{a d}}+\mu_{1} \int_{c}^{d} \frac{\mathrm{d} t}{\pi} \frac{f^{\prime}(t)}{\sqrt{(t-a)(d-t)}}=\mathcal{O}\left(\mathrm{e}^{\alpha / \mu_{1}}\right), \\
& \kappa= \int_{c}^{d} \frac{\mathrm{d} x}{2 \pi} \sqrt{\frac{d-x}{x-a}}\left(1-\frac{\nu}{x} \sqrt{\frac{a}{d}}\right) \\
&+\mu_{1} \int \frac{\mathrm{d} x}{2 \pi} \sqrt{\frac{d-x}{x-a}} \int_{c}^{d} \frac{\mathrm{d} t}{\pi} \sqrt{\frac{t-a}{d-t}} \frac{f^{\prime}(t)}{t-x}+\mathcal{O}\left(\mathrm{e}^{\left.\alpha / \mu_{1} / \mu_{1}\right),}\right. \\
& s= \int_{c}^{d} \frac{f(x) \mathrm{d} x}{2 \pi} \sqrt{\frac{d-x}{x-a}}\left(1-\frac{\nu}{x} \sqrt{\frac{a}{d}}\right) \\
&+\mu_{1} \int \frac{f(x) \mathrm{d} x}{2 \pi} \sqrt{\frac{d}{x-a}} \int_{c}^{d} \frac{\mathrm{d} t}{\pi} \sqrt{\frac{t-a}{d-t}} \frac{f^{\prime}(t)}{t-x}+\mathcal{O}\left(\mathrm{e}^{\left.\alpha / \mu_{1} / \mu_{1}\right) .} .\right.
\end{aligned}
$$


Performing a similar expansion for the second phase, i.e. for $\mu_{1} f^{\prime} \rightarrow 0^{+}$, we obtain the same equations without the $\mathcal{O}\left(\mathrm{e}^{\alpha / \mu_{1}}\right)$ and $\mathcal{O}\left(\mathrm{e}^{\alpha / \mu_{1}} / \mu_{1}\right)$ terms. But these corrections give no contribution to any power series at $\mu_{1}=0$, hence all the derivatives of the energy (related to $\mu_{1}$ via (3.19)) are equal on both sides of the transition. There is a weak non analyticity (an essential singularity) at $\mu_{1}=0$, corresponding to $s=s_{0}(\kappa)$. Using the standard terminology of phase transition, we can say that the Coulomb gas undergoes a phase transition of infinite order at $s=s_{0}(\kappa)$.

Coming back to the case $f(x)=\sqrt{x}$ with $\nu=0$ discussed in the main body of the paper, the discussion above proves the result of Section 3.3.3. Indeed, Eq. (A.32) reduces to

$$
\alpha=2 \pi \sqrt{4-c_{0}} .
$$

To recover Eq. (3.39), we need to rewrite the terms $\mathcal{O}\left(\mathrm{e}^{\alpha / \mu_{1}}\right)$ and $\mathcal{O}\left(\mathrm{e}^{\alpha / \mu_{1}} / \mu_{1}\right)$ in terms of $\epsilon=s-s_{0}$. This can be done using Eq. (A.36), which reduces to

$$
\mu_{1}=-\frac{2 \pi^{2}}{4-c_{0}+c_{0} \ln \left(c_{0} / 4\right)} \epsilon+\mathcal{O}\left(\epsilon^{2}\right)+\mathcal{O}\left(\mathrm{e}^{\alpha / \mu_{1}} / \mu_{1}\right)
$$

where we have kept the subleading $\mathcal{O}\left(\mathrm{e}^{\alpha / \mu_{1}} / \mu_{1}\right)$ as it is the one that differs between Phases 1 and 2 . From this relation, we deduce

$$
\frac{\alpha}{\mu_{1}} \simeq-\frac{\sqrt{4-c_{0}}}{\pi \epsilon}\left(4-c_{0}+c_{0} \ln \frac{c_{0}}{4}\right)=-\frac{\gamma}{\epsilon}, \quad \text { for } \quad \mu_{1} \rightarrow 0
$$

where $\gamma$ is the constant given in Eq. (3.40). We can thus obtain the energy (or equivalently the large deviations function $\Phi_{\kappa}$ ) by using the thermodynamic identity (3.19). Since the only difference between the two phases is the $\mathcal{O}\left(\mathrm{e}^{\alpha / \mu_{1}} / \mu_{1}\right)=\mathcal{O}\left(\mathrm{e}^{-\gamma / \epsilon} / \epsilon\right)$, we have, for $\epsilon>0$ :

$$
\frac{\mathrm{d}}{\mathrm{d} \epsilon}\left[\Phi_{\kappa}\left(s_{0}+\epsilon\right)-\Phi_{\kappa}\left(s_{0}-\epsilon\right)\right]=\mathcal{O}\left(\mathrm{e}^{-\gamma / \epsilon} / \epsilon\right) .
$$

Integrating this last relation yields (3.39).

\section{References}

1. C. W. J. Beenakker, Random-matrix theory of quantum transport, Rev. Mod. Phys. 69(3), 731-808 (1997).

2. T. Guhr, A. Müller-Groeling, and H. A. Weidenmüller, Random-matrix theories in quantum physics: common concepts, Phys. Rep. 299(4/6), 189-425 (1998).

3. I. L. Aleiner, P. W. Brouwer, and L. I. Glazman, Quantum effects in Coulomb blockade, Phys. Rep. 358(5-6), 309-440 (2002).

4. P. A. Mello and N. Kumar, Quantum transport in mesoscopic systems - Complexity and statistical fluctuations, Oxford University Press, 2004.

5. P. W. Brouwer, Generalized circular ensemble of scattering matrices for a chaotic cavity with nonideal leads, Phys. Rev. B 51, 16878-16884 (1995).

6. P. A. Mello and H. U. Baranger, Interference phenomena in electronic transport through chaotic cavities: An information-theoretic approach, Waves Random Media 9, 105-162 (1999)

7. H.-J. Sommers, W. Wieczorek, and D. V. Savin, Statistics of conductance and shot noise power for chaotic cavities, Acta Phys. Pol. A 112, 691 (2007)

8. P. Vivo, S. N. Majumdar, and O. Bohigas, Distributions of Conductance and Shot Noise and Associated Phase Transitions, Phys. Rev. Lett. 101, 216809 (2008).

9. B. A. Khoruzhenko, D. V. Savin, and H.-J. Sommers, Systematic approach to statistics of conductance and shot-noise in chaotic cavities, Phys. Rev. B 80, 125301 (2009). 
10. P. Vivo, S. N. Majumdar, and O. Bohigas, Probability distributions of Linear Statistics in Chaotic Cavities and associated phase transitions, Phys. Rev. B 81, 104202 (2010).

11. P. Vivo and E. Vivo, Transmission eigenvalue densities and moments in chaotic cavities from random matrix theory, J. Phys. A: Math. Theor. 41(12), 122004 (2008).

12. A. Grabsch and C. Texier, Capacitance and charge relaxation resistance of chaotic cavities - Joint distribution of two linear statistics in the Laguerre ensemble of random matrices, Europhys. Lett. 109, 50004 (2015).

13. F. D. Cunden, P. Facchi, and P. Vivo, Joint statistics of quantum transport in chaotic cavities, Europhys. Lett. 110, 50002 (2015).

14. D. N. Page, Average entropy of a subsystem, Phys. Rev. Lett. 71, 1291-1294 (1993).

15. P. Facchi, U. Marzolino, G. Parisi, S. Pascazio, and A. Scardicchio, Phase Transitions of Bipartite Entanglement, Phys. Rev. Lett. 101, 050502 (2008).

16. A. De Pasquale, P. Facchi, G. Parisi, S. Pascazio, and A. Scardicchio, Phase transitions and metastability in the distribution of the bipartite entanglement of a large quantum system, Phys. Rev. A 81, 052324 (2010).

17. P. Facchi, G. Florio, G. Parisi, S. Pascazio, and K. Yuasa, Entropy-driven phase transitions of entanglement, Phys. Rev. A 87, 052324 (2013).

18. C. Nadal, S. N. Majumdar, and M. Vergassola, Phase Transitions in the Distribution of Bipartite Entanglement of a Random Pure State, Phys. Rev. Lett. 104, 110501 (2010).

19. C. Nadal, S. N. Majumdar, and M. Vergassola, Statistical Distribution of Quantum Entanglement for a Random Bipartite State, J. Stat. Phys. 142(2), 403-438 (2011).

20. C. Nadal and S. N. Majumdar, Nonintersecting Brownian interfaces and Wishart random matrices, Phys. Rev. E. 79, 061117 (2009).

21. C. Nadal, Matrices aléatoires et leurs applications à la physique statistique et physique quantique, PhD thesis, Université Paris-Sud, 2011.

22. S. N. Majumdar and G. Schehr, Top eigenvalue of a random matrix: large deviations and third order phase transition, J. Stat. Mech. 2014(1), P01012 (2014).

23. F. J. Dyson, Statistical Theory of the Energy Levels of Complex Systems. I, J. Math. Phys. 3(1), 140-156 (1962) ; ibid 3(1), 157-165 (1962) ; ibid 3(1), 166-175 (1962).

24. G. Ben Arous and A. Guionnet, Large deviations for Wigner's law and Voiculescu's non-commutative entropy, Prob. Theo. Relat. Fields 108(4), 517-542 (1997).

25. G. Ben Arous and O. Zeitouni, Large deviations from the circular law, ESAIM: Prob. Stat. 2, 123-134 (1998).

26. E. Brézin, C. Itzykson, G. Parisi, and J. B. Zuber, Planar diagrams, Communications in Mathematical Physics 59(1), 35-51 (1978).

27. D. S. Dean and S. N. Majumdar, Large Deviations of Extreme Eigenvalues of Random Matrices, Phys. Rev. Lett. 97, 160201 (2006).

28. D. S. Dean and S. N. Majumdar, Extreme value statistics of eigenvalues of Gaussian random matrices, Phys. Rev. E 77, 041108 (2008).

29. P. Vivo, S. N. Majumdar, and O. Bohigas, Large deviations of the maximum eigenvalue in Wishart random matrices, J. Phys. A: Math. Theor. 40(16), 4317 (2007).

30. S. N. Majumdar, C. Nadal, A. Scardicchio, and P. Vivo, Index distribution of Gaussian random matrices, Phys. Rev. Lett. 103, 220603 (2009).

31. S. N. Majumdar, C. Nadal, A. Scardicchio, and P. Vivo, How many eigenvalues of a Gaussian random matrix are positive?, Phys. Rev. E 83, 041105 (2011).

32. R. Marino, S. N. Majumdar, G. Schehr, and P. Vivo, Index distribution of Cauchy random matrices, J. Phys. A: Math. Theor. 47, 055001 (2014).

33. S. N. Majumdar and P. Vivo, Number of Relevant Directions in Principal Component Analysis and Wishart Random Matrices, Phys. Rev. Lett. 108, 200601 (2012). 
34. R. Marino, S. N. Majumdar, G. Schehr, and P. Vivo, Phase Transitions and Edge Scaling of Number Variance in Gaussian Random Matrices, Phys. Rev. Lett. 112, 254101 (2014).

35. R. Marino, S. N. Majumdar, G. Schehr, and P. Vivo, Number statistics for $\beta$-ensembles of random matrices: applications to trapped fermions at zero temperature, Phys. Rev. E 94, 032115 (2016).

36. C. Texier and S. N. Majumdar, Wigner Time-Delay Distribution in Chaotic Cavities and Freezing Transition, Phys. Rev. Lett. 110, 250602 (2013).

37. J. Wishart, The Generalised Product Moment Distribution in Samples from a Normal Multivariate Population, Biometrika 20A(1-2), 32-52 (1928).

38. M. E. Fisher, Walks, Walls, Wetting, and Melting, J. Stat. Phys. 34, 667 (1984).

39. T. L. Einstein, Applications of Ideas from Random Matrix Theory to Step Distributions on "Misoriented" Surfaces, Annales Henri Poincaré 4(2), 811-824 (2003).

40. P. G. de Gennes, Soluble Model for Fibrous Structures with Steric Constraints, J. Chem. Phys. 48(5), 2257-2259 (1968).

41. F. J. Dyson, The Threefold Way. Algebraic Structure of Symmetry Groups and Ensembles in Quantum Mechanics, J. Math. Phys. 3, 1199-1215 (1962).

42. F. G. Tricomi, Integral equations, Interscience, London, 1957.

43. F. D. Cunden, P. Facchi, and P. Vivo, A shortcut through the Coulomb gas method for spectral linear statistics on random matrices, J. Phys. A: Math. Theor. 49, 135202 (2016).

44. A. Grabsch and C. Texier, Distribution of spectral linear statistics on random matrices beyond the large deviation function - Wigner time delay in multichannel disordered wires, J. Phys. A: Math. Theor. 49, 465002 (2016).

45. V. A. Marčenko and L. A. Pastur, Distribution of eigenvalues for some sets of random matrices, Math. USSR-Sb 1(4), 457 (1967).

46. I. S. Gradshteyn and I. M. Ryzhik, Table of integrals, series and products, 5th Edition (Academic Press), 1994.

47. K. Johansson, Shape fluctuations and random matrices, Commun. Math. Phys. 209 437-476 (2000).

48. I. M. Johnstone, On the distribution of the largest eigenvalue in principal component analysis, Ann. Statist. 29(2), 295-327 (2001).

49. S. N. Majumdar and M. Vergassola, Large deviations of the maximum eigenvalue for Wishart and Gaussian random matrices, Phys. Rev. Lett. 102, 060601 (2009).

50. K. Damle, S. N. Majumdar, V. Tripathi, and P. Vivo, Phase transitions in the distribution of the Andreev conductance of superconductor-metal junctions with multiple transverse modes, Phys. Rev. Lett. 107, 177206 (2011).

51. R. Allez, J. Touboul, and G. Wainrib, Index distribution of the Ginibre ensemble, J. Phys. A: Math. Theor. 47, 042001 (2014).

52. F. D. Cunden, A. Maltsev, and F. Mezzadri, Fluctuations in the two-dimensional onecomponent plasma and associated fourth-order phase transition, Phys. Rev. E 91, 060105 (2015).

53. M. L. Mehta, Random matrices, Elsevier, Academic, New York, third edition, 2004.

54. P. J. Forrester, Log-gases and random matrices, Princeton University Press, 2010.

55. L. W. Cheuk, M. A. Nichols, M. Okan, T. Gersdorf, V. V. Ramasesh, W. S. Bakr, T. Lompe, and M. W. Zwierlein, Quantum-Gas Microscope for Fermionic Atoms, Phys. Rev. Lett. 114, 193001 (2015).

56. E. Haller, J. Hudson, A. Kelly, D. A. Cotta, B. Peaudecerf, G. D. Bruce, and S. Kuhr, Single-atom imaging of fermions in a quantum-gas microscope, Nat. Phys. 11, 738-742 (2015).

57. M. F. Parsons, F. Huber, A. Mazurenko, C. S. Chiu, W. Setiawan, K. Wooley-Brown, S. Blatt, and M. Greiner, Site-Resolved Imaging of Fermionic ${ }^{6} \mathrm{Li}$ in an Optical Lattice, Phys. Rev. Lett. 114, 213002 (2015). 